NISTIR 8111

\title{
Optimization of Registration Performance Metrics
}

\author{
Marek Franaszek \\ Geraldine S. Cheok
}

This publication is available free of charge from:

http://dx.doi.org/10.6028/NIST.IR.8111

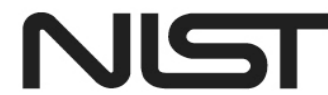

National Institute of 
NISTIR 8111

\title{
Optimization of Registration Performance Metrics
}

\author{
Marek Franaszek \\ Geraldine S. Cheok \\ Intelligent Systems Division \\ Engineering Laboratory
}

This publication is available free of charge from:

http://dx.doi.org/10.6028/NIST.IR.8111

February 2016

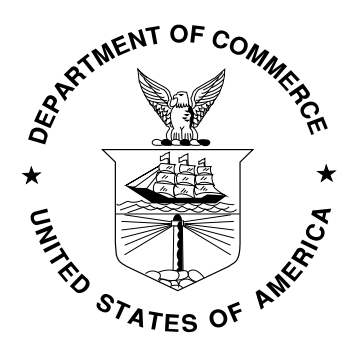

U.S. Department of Commerce Penny Pritzker, Secretary

National Institute of Standards and Technology Willie May, Under Secretary of Commerce for Standards and Technology and Director 


\section{Contents}

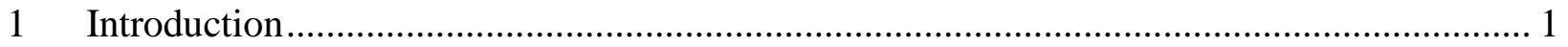

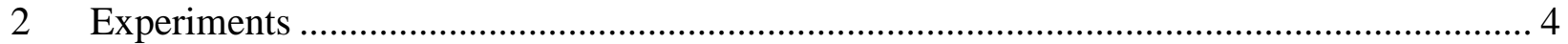

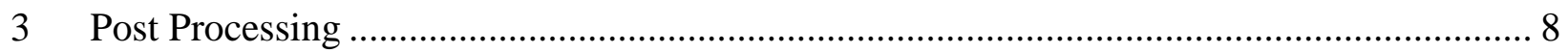

3.1 Characterization of instrument noise...................................................................... 8

3.2 Check rigid body assumption for registration ............................................................... 8

3.3 Metrics for quality of registration ........................................................................... 9

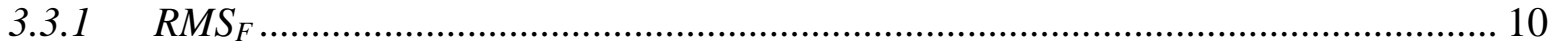

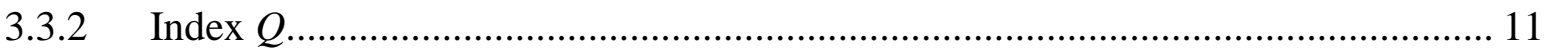

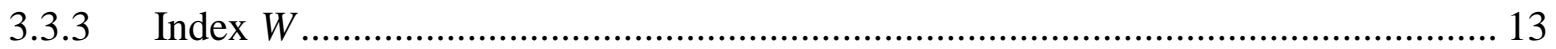

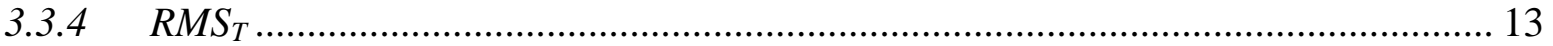

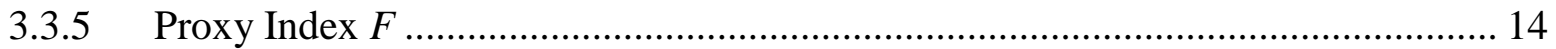

3.3.6 Overview of metrics.......................................................................................... 15

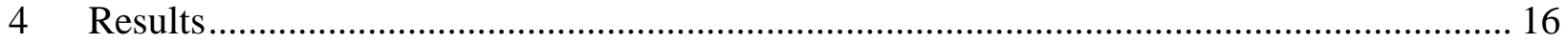

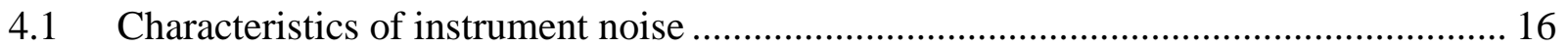

4.2 Check of rigid body assumption.............................................................................. 18

4.3 Calculations of different registration performance metrics.............................................. 20

4.3.1 Performance metrics for registration with $N=4$ fiducials.................................... 21

4.3.2 Effect of the number of fiducials on registration performance............................... 29

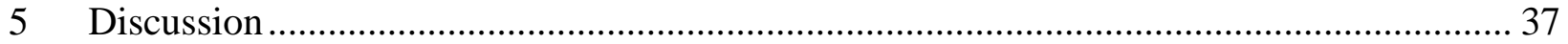

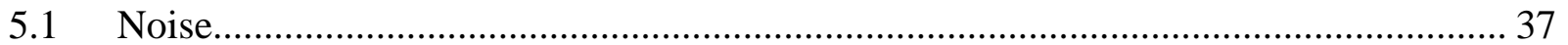

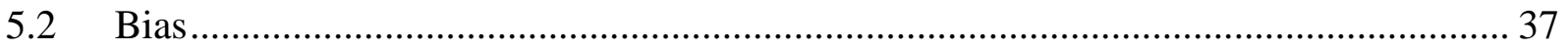

5.3 Performance metrics for registration................................................................................ 38

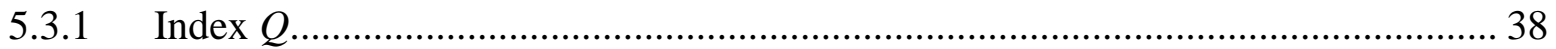

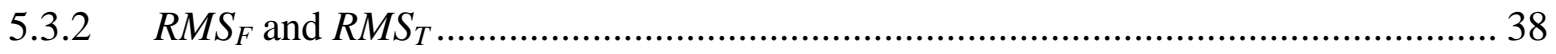

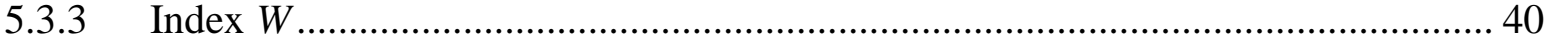

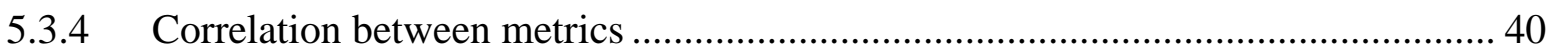

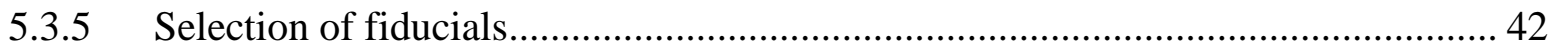

5.3.6 Effect of additional fiducials.............................................................................. 42

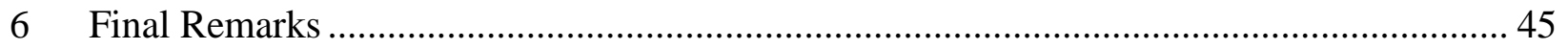

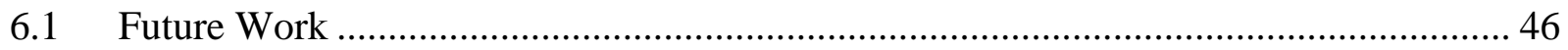




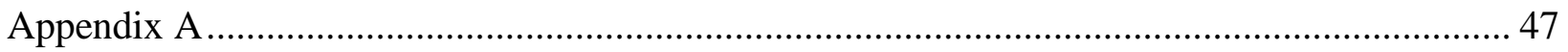

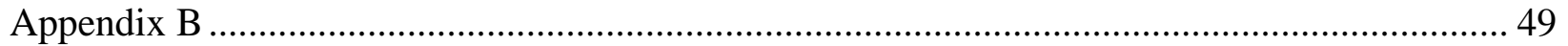

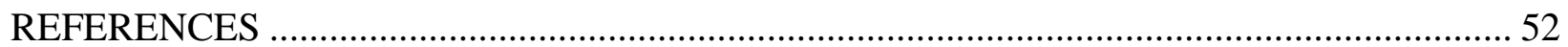




\section{DISCLAIMER}

Certain trade names and company products are mentioned in the text or identified in an illustration in order to adequately specify the experimental procedure and equipment used. In no case does such an identification imply recommendation or endorsement by the National Institute of Standards and Technology, nor does it imply that the products are necessarily the best available for the purpose. 


\begin{abstract}
Perception systems are a very important component of automation and robotics. These systems are used to localize objects in a local coordinate frame. The data typically have to be available for use in another coordinate frame, which requires that the data be transformed from one coordinate frame to another. There are different methods to obtain the transformation. In this study, rigid body, point-based registration is investigated. The report details the effort to develop guidelines for this type of registration based on physical experiments and noise characterization. The work also involves the development of four performance metrics to quantify the quality of the registration and guidance to select the number of points and their locations for optimal registration. Three of the proposed performance metrics give the user an estimate of the distance between a transformed point and its expected true location, and the fourth metric gives the user an estimate of the uncertainty of the transformed point.

Based on the experimental results, an index is proposed to aid a user in selecting fiducials so that the registration is optimized. This optimization depends on the noise characteristics of the instruments used and the performance metric chosen. The results also indicate that adding more fiducials may not always be beneficial. Additionally, it was shown that the quality of registration was adversely affected by the presence of bias.
\end{abstract}




\section{INTRODUCTION}

The National Institute of Standards and Technology has a project titled Tools for Collaborative Robots within SME (small and medium enterprise) Workcells within the Robotic Systems for Smart Manufacturing program. The objective of this project is to deliver a suite of tools that facilitates calibration procedures for individual robots, robot-to-robot coordination, sensors, and grippers to mitigate the lack of automation and technical expertise that currently prevent small and medium-sized manufacturers from adopting robotic systems.

Typically, sensors record positional measurements in their own local coordinate frame. When measuring with line-of-sight instruments, the instrument may have to be moved to different locations due to occlusions and the data will have to be registered so that they are in the same coordinate frame. For robotic systems to use the positional data from an external sensor, the robot and the data have to use the same coordinate frame; that is, the coordinate frame of the robot must be registered with the coordinate frame of the sensor used to collect the data, or vice versa, or the coordinate frames of the robot and the sensor must both be registered to a global coordinate frame.

The purpose of registration is to obtain the transformation matrix between two coordinate frames. In this report, the working frame is the one to be transformed and the reference frame is the frame to which the working frame is transformed. Registration is needed when some points are measured only in the working frame but are needed in the reference frame. Then, the transformation matrix is used to transform the points measured in the working frame to the reference frame. In order to calculate the transformation matrix, points measured in both coordinate frames are required; these are called fiducial points in this report.

A measurement is considered good if it is accurate and precise. Accuracy is the closeness of agreement between a measured quantity and the true value [1]. Accuracy is affected by systematic error and precision is affected by random error. Accuracy is quantified by the magnitude of the measurement error which is defined as a measurement minus ground truth. In general, ground truth involves obtaining the measurand with a reference instrument whose error is at least an order of magnitude less than the instrument being used. For registration, the measurand is a set of transformation parameters: three rotation angles and three components of

translation. Since one cannot physically place and orient the coordinate frames of two instruments so that they align perfectly, the ground truth measurands cannot be obtained. Therefore, the error of registration cannot be determined. In order to quantify the quality of registration, other metrics have been used. Often, the root mean square, $R M S_{F}$, of the distances between transformed fiducials and the corresponding fiducials in the reference frame is used as a substitute metric, see Figure 1.1. 


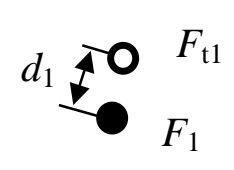

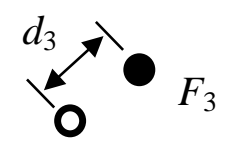

$F_{\mathrm{t} 3}$
$F_{\mathrm{i}}=$ Fiducial $i$ in reference frame

$F_{\mathrm{ti}}=$ Fiducial $i$ in working frame transformed to reference frame

$$
R M S_{F}=\sqrt{\frac{\sum_{i=1}^{n} d_{i}^{2}}{n}}=\sqrt{\frac{d_{1}^{2}+d_{2}^{2}+d_{3}^{2}}{3}}
$$

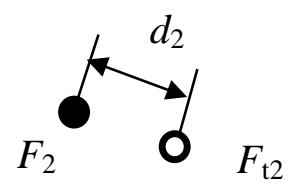

Figure 1.1. Commonly used metric for registration error

Precision is the closeness of agreement between measured values obtained by replicate measurements under specified conditions [1] and can be quantified by the uncertainty of the measurement, which can be represented by the standard deviation of repeated measurements. For registration, uncertainty can be obtained from the covariance matrix of the six registration parameters. Better registration is indicated by smaller values of uncertainties. However, knowing the uncertainties of the registration parameters does not readily allow a user to determine the uncertainty of a point transformed from the working to the reference frame. For example, if the uncertainty of the rotation angles is $20 \mathrm{mrad}$, what does this mean and is this acceptable? Therefore, for practical applications, a more useful measure of the uncertainty would provide the uncertainty of a point to which the registration transformation was applied.

Performance of point-based registration has been studied for a long time [2-9]. In the literature, three main characteristics were investigated: Fiducial Localization Error (FLE), Fiducial Registration Error (FRE), and Target Registration Error (TRE). FLE is the distance between the unknown true location of a fiducial and its measured location and thus, cannot be measured. FRE is the root mean square of the distances between transformed fiducials and the corresponding fiducials in the reference frame (equivalent to $R M S_{F}$ schematically shown in Figure 1.1). TRE is the distance between a transformed target and its corresponding point in the reference frame. A target is a point which is usually measured only in the working frame but not in the reference frame; thus, a target point is not used to register two coordinate systems but is a point of interest for the application. For the researchers in [2-9] the metric of critical importance 
is the TRE. Therefore, there has been some research building on Fitzpatrick et al.'s work [4] that focused on relating TRE with FRE and analytical formulations supported by numerical simulations were obtained [10-14]. However, more recent studies show that FRE cannot be used as an estimate of TRE and, in fact, both are statistically uncorrelated [12, 15-19]. While some experimental studies have been reported earlier [20-22], most of the published results were obtained based on computer simulations.

This report presents results from extensive experimental studies in which three different instruments were used to collect 3D data. The experimental conditions represent actual or field situations which cannot be accurately modeled in computer simulations. For example, in our study, noise magnitude at different fiducial locations varies substantially and one instrument has a large bias in the collected data. As a consequence, we show that the placement of fiducial points affects the value of $R M S_{F}$, contrary to some of the theoretical results supported by numerical simulations.

The results of the experiments are used to develop guidelines for sensor-to-sensor registration procedures. This task utilizes a tool previously developed in the project: propagation of random error (noise) from registration parameters to the transformed data. These guidelines will aid SMEs in determining the number and placement of registration points to minimize registration error based on instrument noise. In addition, metrics were developed to give SMEs some indication as to the quality of the registration.

The report is organized as follows: the experimental plan and data collection are in Chapter 2, the post-processing of the data in Chapter 3, results in Chapter 4, a discussion of the results in Chapter 5, and a summary and conclusions are provided in Chapter 6 . 


\section{EXPERIMENTS}

The basic concept for the experiments was to collect a series of points (fiducials) and to use subsets of these points to determine the required number and placement of the fiducials to minimize registration error. It was decided that the points would be collected in a grid pattern and that a $5 \times 5 \times 5$ grid would yield a sufficiently dense dataset and still be practical in terms of data collection as the data was to be collected in one day. The reason for the one day criterion was to avoid re-calibration of the instruments if the data collection were to span multiple days. It was expected that the re-calibration process would result in minor differences in the day-to-day measurements. Since the instrument noise is on the order of micrometers, even minor differences in the measurements could be significant and cause the results to be confounded.

The data were collected using three instruments, each having a different noise characteristic: a laser tracker (LT), a motion capture system (System A), and a large-scale metrology system (System B). The measurement uncertainties are $\pm 25 \mu \mathrm{m}$ for the laser tracker, $\pm 250 \mu \mathrm{m}$ for the large-scale metrology system, while the accuracy of the motion capture system is only specified as sub-millimeter. To acquire measurements with these instruments, different accessories were required. The laser tracker uses a spherical mounted retroreflector (SMR) to obtain measurements to the center of the SMR. The large-scale metrology system uses a vector bar containing two detectors to obtain measurements. The motion capture system consists of six cameras and requires the use of spherical reflective markers to obtain measurements.

For the experiments described in this report, it was essential that the same point be measured by the three different metrology systems. To achieve this criterion, a vector bar as shown in Figure 2.1a was used. On top of the vector bar is a magnetic nest for a $12.7 \mathrm{~mm}$ diameter sphere. A $12.7 \mathrm{~mm}$ diameter SMR or reflective marker can then be placed in the nest which enables measurements to the center of the SMR or sphere to be made by the laser tracker or the motion capture system. The vector bar contains two detectors centered inside the black regions of the vector bar. The distance $(D)$ between the top detector and the center of a $12.7 \mathrm{~mm}$ sphere located in the nest (see Figure 2.1c) is known and supplied by the vendor.

For the large-scale metrology system, 200 measurements of the vector bar were obtained. These measurements include the 3D locations of the two detectors. To obtain the location of a $12.7 \mathrm{~mm}$ sphere as measured by the large-scale metrology system, a line was determined by the two detectors, and the location of the center of the sphere in the nest was then equal to the point along the line offset by the distance $D$ from the top detector. To obtain the location of the sphere with the motion capture system, a $12.7 \mathrm{~mm}$ diameter reflective marker was placed in the nest on top of the vector bar and at least 200 measurements were recorded, see Figure 2.1b.

To obtain the location of the sphere with a laser tracker, a $12.7 \mathrm{~mm}$ diameter SMR was placed in the nest and one measurement was made, see Figure 2.1c. Only one measurement was made because the setting for the laser tracker was such that the recorded measurement was the average 
of 50 measurements. A separate experiment consisting of measuring 15 locations throughout the work volume where 20 repeats were made at each location showed that the average instrument noise was $1.7 \mu \mathrm{m}$ (standard deviation of the data was $0.9 \mu \mathrm{m}$ ).

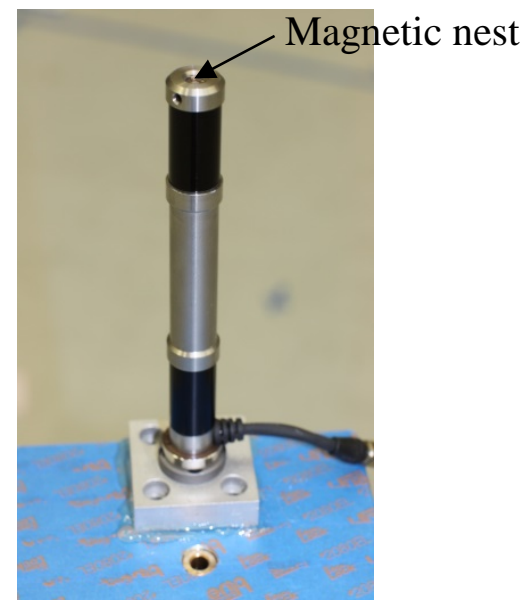

a. Vector bar.

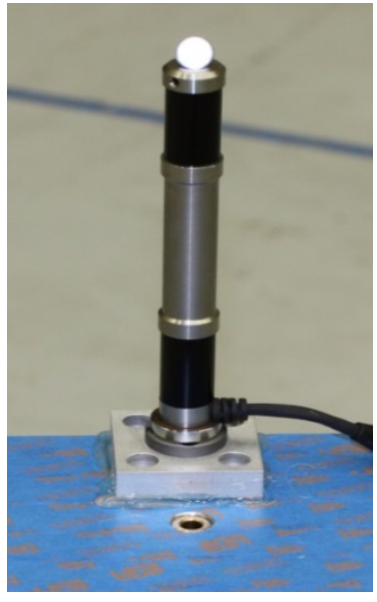

b. $12.7 \mathrm{~mm}$ diameter reflective marker in the magnetic nest.

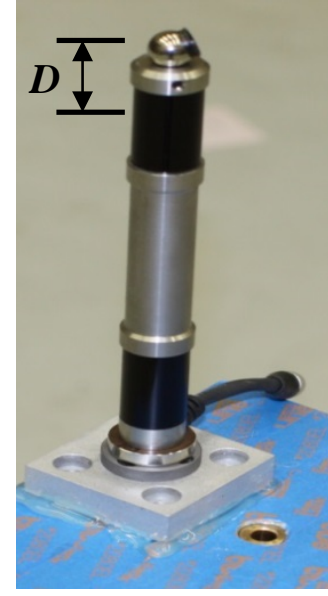

c. $12.7 \mathrm{~mm}$ diameter SMR in the magnetic nest.

Figure 2.1. Measurement of the "same” point using a vector bar.

The size of the space (or work volume) to obtain the points was governed by the motion capture system. The dimensions of the work volume where measurements were obtained were approximately $3 \mathrm{~m} \mathrm{x} 3 \mathrm{~m}$ x $1.8 \mathrm{~m}$ ( $\mathrm{L} \mathrm{x} \mathrm{W} \mathrm{x} \mathrm{H})$. The vector bar attached to a metrology stand in the work space is shown in Figure 2.2 where the blue tape delineates the extents of the work volume. 


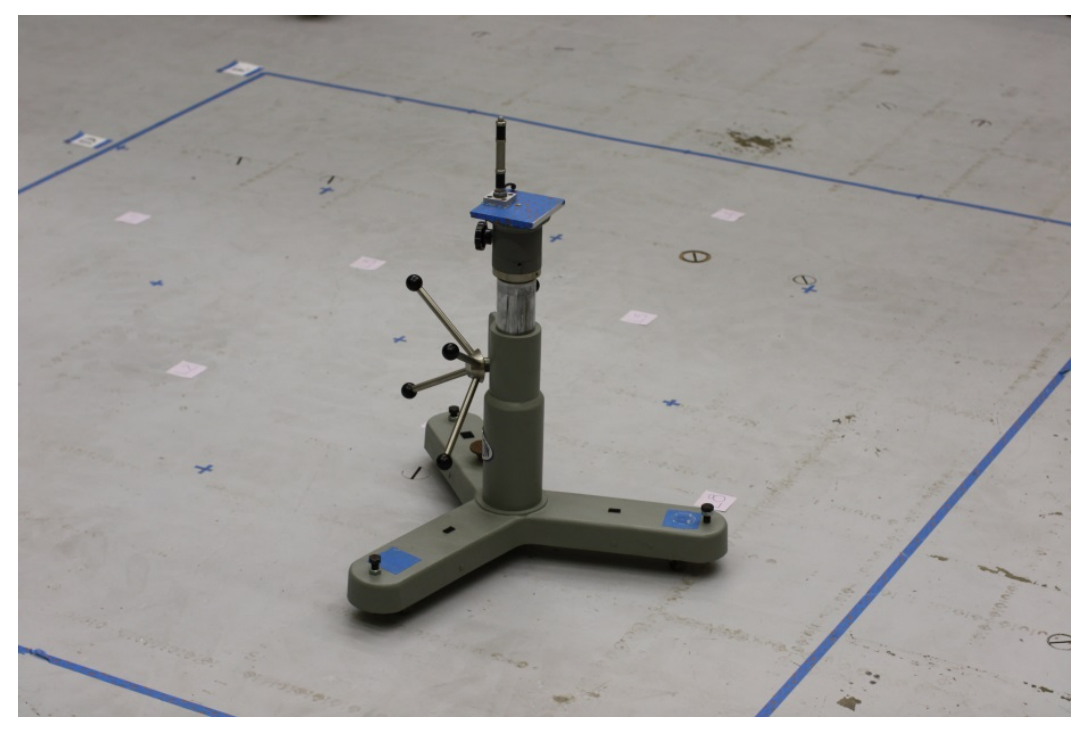

Figure 2.2. Vector bar on metrology stand in work volume.

As stated earlier, the data was measured in a nominal $5 \times 5 \times 5$ grid pattern. Marks were made on the floor within the work volume to indicate the approximate "intersection" of the grid lines where measurements were to be obtained. The grid spacing was not uniform as uniformity was not critical and the heights of two of the layers were dictated by the available metrology stands. Since uniformity was not critical, no effort was made beyond allowing for the visual centering of the metrology stand over a mark on the floor, i.e., no additional aids were developed to help in centering the stand over a mark. The elimination of the need to ensure that the stand is centered precisely over a mark on the floor, that the stand is level, and that the vector bar is in the same position relative to the center of the stand (since the vector bar is offset from the center of the stand), made the collection of the data in one day feasible.

For the data collection, the vector bar was attached to a plate which was attached to a metrology stand, except for the lowest horizontal layer. For the lowest horizontal layer, the plate with the vector bar was placed directly on the floor. To collect the data, the metrology stand was placed at a location, and the data from all three instruments were collected before moving the stand to another location. All data in one layer were collected before the data in another layer were collected. This meant that since the stands were visually centered, the location of Point A in one horizontal layer may be slightly shifted in the horizontal plane from Point A in another horizontal layer.

The 5 x 5 x 5 grid pattern yielded $M=125$ measurements. The 125 points make up the set of points from which different combinations of $N$ points were selected and used to register one instrument to another. This set of points serves as fiducial points. In addition to the fiducial points, 16 other points were measured throughout the work volume. These 16 points were not 
used in the registration process but were used to determine the quality of the registration (see Section 3.3) and will be called test points. Typically, in practical applications, the quality of registration is based solely on the fiducials and test points are not used. However, in this report, to quantify the registration process, test points were measured by all three instruments (System A, B, and LT) because, in actual applications, it is the distance errors of test points (points not used for registration) that are important.

Figure 2.3 shows the relative locations of the 125 fiducial points and the 16 test points as measured with the laser tracker. For easier visualization, the different horizontal layers of the grid points are shown in different colors (red or GND layer is about ground level) in Figure 2.3a, and the test points are shown in black in Figure 2.3b.

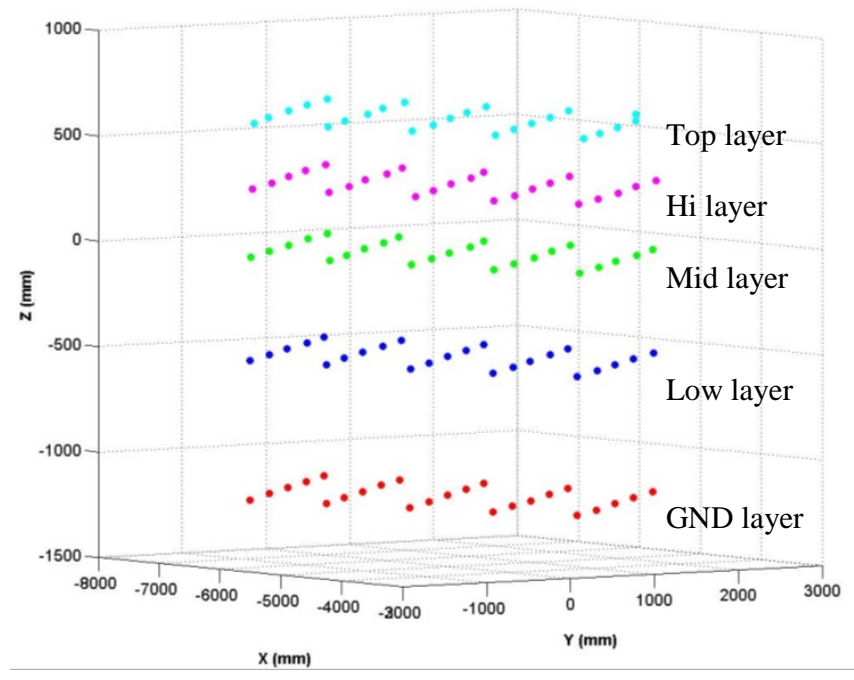

a. Fiducial points as measured with the laser tracker.

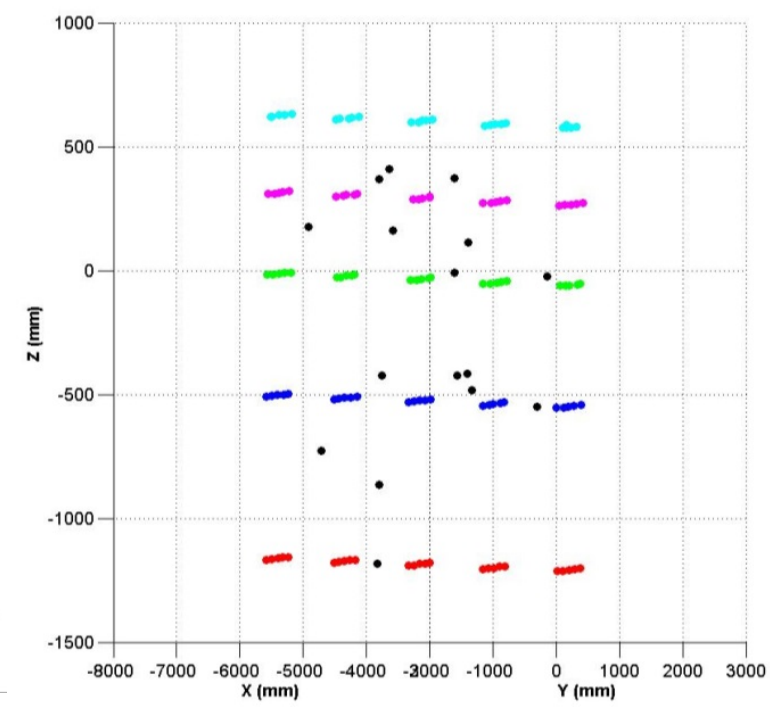

b. Test points (black) relative to the grid points.

Figure 2.3. Relative locations of fiducial and test points. 


\section{POST PROCESSING}

In this study, 3D rigid body registration was used to register the points acquired in two different coordinate frames. Obviously, this type of registration assumes that the distance between the same pair of fiducial points does not depend on which instrument performs the measurement. Also, the quality of the registration depends on the:

1. spatial distribution of the fiducial points,

2. magnitude of the instrument noise at the fiducial points,

3. whether the magnitude of the noise throughout the work volume is constant or variable.

Therefore, data processing was performed to achieve three main objectives: 1 ) to characterize the noise of the instruments used in the experiment; 2) to check the validity of the rigid body assumption on which the registration procedure is based; 3) to compare different performance metrics which gauge the quality of the registration.

In post processing the data, two methods were used. In method 1, the fiducial and test points were first averaged over the 200 repeat measurements. Then, registration and other performance metrics (like root mean square of distances) were calculated using these averaged points. This averaging, basically, removes the effect of noise from the results obtained using method 1. In method 2, registration was performed for each repeated measurement. Thus, in the second method, 200 registrations were performed for a given nominal set of fiducials and for each individual registration, the corresponding performance metrics were calculated. Finally, for each metric, the mean and variance of the 200 values were obtained. The results presented in the subsequent sections were obtained using method 1, unless otherwise noted.

\subsection{CHARACTERIZATION OF INSTRUMENT NOISE}

For each $M=125$ fiducial points and $K=16$ test points, the corresponding average and 3x3 covariance matrix was calculated from the 200 repeated measurements acquired by Systems A and $\mathrm{B}$. The diagonal elements of the covariance matrix are the variances of the 3D coordinates $[\operatorname{var}(x), \operatorname{var}(y), \operatorname{var}(z)]$ while the three off-diagonal elements indicate the cross-correlation between the coordinates. The magnitude of noise, $\sigma_{0}$, is defined by the following equation:

$$
\sigma_{0}(x, y, z)=\sqrt{\operatorname{var}(x)+\operatorname{var}(y)+\operatorname{var}(z)} .
$$

\subsection{CHECK RIGID BODY ASSUMPTION FOR REGISTRATION}

To verify the rigid body assumption, the distance $d_{i, j}$ was calculated between a pair of fiducial points $(i, j)$, where the coordinates of the points were the averages of the 200 measurements, obtained with Systems A or B. These distances were then compared with the corresponding 
distances $D_{\mathrm{i}, \mathrm{j}}$ obtained with LT. For this check, the laser tracker measurements were used as the noise free, ground truth distances, i.e., $\operatorname{var}\left(D_{\mathrm{i}, \mathrm{j}}\right)=0$.

Under ideal conditions, distances, $d_{\mathrm{i}, \mathrm{j}}$ and $D_{\mathrm{i}, \mathrm{j}}$, should be equal within some measurement error.

In reality, the rigid body assumption does not perfectly hold and the error $L$ between $d_{\mathrm{i}, \mathrm{j}}$ and $D_{\mathrm{i}, \mathrm{j}}$, and its variance $\operatorname{var}(L)$ are calculated as follows

$$
\begin{aligned}
& L\left(x_{i}, y_{i}, z_{i}, x_{j}, y_{j}, z_{j}\right)=d_{i, j}-D_{i, j} \\
& d_{i, j}\left(x_{i}, y_{i}, z_{i}, x_{j}, y_{j}, z_{j}\right)=\sqrt{\left(x_{i}-x_{j}\right)^{2}+\left(y_{i}-y_{j}\right)^{2}+\left(z_{i}-z_{j}\right)^{2}} \\
& \operatorname{var}(L)=J C_{i, j} J^{T} \\
& p=L / \sqrt{\operatorname{var}(L)},
\end{aligned}
$$

where the Jacobian vector $\boldsymbol{J}$ equals

$$
\boldsymbol{J}\left(x_{i}, y_{i}, z_{i}, x_{j}, y_{j}, z_{j}\right)=\frac{1}{d_{i, j}}\left[x_{i}-x_{j}, y_{i}-y_{j}, z_{i}-z_{j}, x_{j}-x_{i}, y_{j}-y_{i}, z_{j}-z_{i}\right] \text {, }
$$

$\boldsymbol{J}^{T}$ is the transposed vector and the $6 \times 6$ covariance matrix $\boldsymbol{C}_{i, j}$ is

$$
C_{i, j}=\left[\begin{array}{cc}
c_{i} & 0 \\
0 & c_{j}
\end{array}\right]
$$

where $\boldsymbol{c}_{i}$ and $\boldsymbol{c}_{j}$ are the $3 \times 3$ covariance matrices at $i^{\text {th }}$ and $j^{\text {th }}$ fiducial locations assuming no cross correlation between them. The ratio $p$ is used to determine if $L$ is within one standard deviation of the noise. A large value of $p$ could indicate the presence of bias.

\subsection{METRICS FOR QUALITY OF REGISTRATION}

The absolute orientation problem (3D rigid body registration) is solved by finding the rotation $\boldsymbol{\Omega}$ and the translation $\boldsymbol{\tau}$ which transforms a set of $N$ fiducial points from one coordinate frame to another by minimizing the error $h$

$$
h=\frac{1}{N} \sum_{n=1}^{N}\left\|\mathbf{\Omega} Z_{n}+\boldsymbol{\tau}-\boldsymbol{Y}_{n}\right\|^{2},
$$

where $\left\{Z_{n}\right\}$ and $\left\{Y_{n}\right\}$ are two sets of corresponding fiducial points in the working and reference frames, respectively.

A standard procedure is to translate the origins of both coordinate frames to the corresponding centroids $\boldsymbol{Z}_{c t r}$ and $\boldsymbol{Y}_{c t r}$ so that $\boldsymbol{z}_{n}=\boldsymbol{Z}_{n}-\boldsymbol{Z}_{c t r}$ and $\boldsymbol{y}_{n}=\boldsymbol{Y}_{n}-\boldsymbol{Y}_{c t r}$. Then, the 3x3 matrix $\boldsymbol{E}$ is calculated as 


$$
\boldsymbol{E}=\left[\boldsymbol{z}_{1}, \mathbf{z}_{2}, \cdots, \mathbf{z}_{N}\right]\left[\boldsymbol{y}_{1}, \boldsymbol{y}_{2}, \cdots, \boldsymbol{y}_{N}\right]^{T}
$$

and its singular value decomposition (SVD) is performed

$$
\boldsymbol{E}=\boldsymbol{U} \boldsymbol{S} \boldsymbol{V}^{T} .
$$

Then, the rotation matrix $\boldsymbol{\Omega}$ can be calculated as

$$
\mathbf{\Omega}=\boldsymbol{V} \boldsymbol{s} \boldsymbol{U}^{T},
$$

where $s$ is equal to

$$
\boldsymbol{S}=\left[\begin{array}{ccc}
1 & 0 & 0 \\
0 & 1 & 0 \\
0 & 0 & \operatorname{det}\left(\boldsymbol{V} \boldsymbol{U}^{T}\right)
\end{array}\right] .
$$

Finally, the translation vector $\tau$ between the centers of the two coordinate frames can be expressed as

$$
\boldsymbol{\tau}=\boldsymbol{Y}_{c t r}-\boldsymbol{\Omega} \boldsymbol{Z}_{c t r} .
$$

\subsection{1 $R M S_{F}$}

When it comes to evaluating the quality of registration, there is no universally accepted metric. The most commonly used metric is the Root Mean Square $\left(R M S_{F}\right)$ of the distances between the transformed fiducials from the working frame and the corresponding fiducials in the reference frame

$$
R M S_{F}=\sqrt{h_{\min }} .
$$

This intuitive and simple measure has a few important deficiencies. First, recall that in this study, the fiducials are the averages of 200 repeats and the repeated measurements enable us to obtain the covariance matrices at those points. In most practical applications, repeated measurements of fiducial points are not performed. Therefore, the $R M S_{F}$ is calculated from a single measurement and its value will likely change due to instrument noise (i.e., if the fiducials were re-measured, the $R M S_{F}$ would be different) especially for a small number of fiducials, $N$. Without the knowledge of the covariance of the fiducials, the uncertainty or magnitude of the variation of $R M S_{F}$ cannot be estimated.

Second, the $R M S_{F}$ is based solely on the fiducial points. As mentioned previously, the test points are important because they represent points of interest and the registration transformation is applied to them. Once $\{\boldsymbol{\Omega}, \boldsymbol{\tau}\}$ is determined from a given set of fiducials, the $R M S_{T}$ (equivalent to TRE in [2-9]) based on the transformed test points in the working frame and the corresponding test points in the reference frame should be calculated; in ideal conditions, $R M S_{T}$ should be zero. 
Third, registration quality depends on two independent factors: 1) performance of the instrument used to collect data for registration (instrument noise); 2) placement of the fiducials in the working volume. The first factor can be determined and depends on the quality of the instrument used. The second factor depends on the experience and knowledge of the operator in selecting the fiducial locations and, thus, may be subject to optimization. $R M S_{F}$ does not differentiate between these two factors.

As stated in the Introduction, good registration should be accurate and precise. The metric $R M S_{F}$ addresses to some extent the accuracy but does not give any information about the precision of the registration. Therefore, several performance metrics to gauge the accuracy and precision of registration are proposed and are presented in the remainder of this section.

\subsubsection{Index $Q$}

Once the transformation $\{\boldsymbol{\Omega}, \boldsymbol{\tau}\}$ is calculated and applied to the test points in the working frame $\boldsymbol{t}_{k}=\left[x_{k}, y_{k}, z_{k}\right]$, the transformed test points in the reference frame, $\left\{\tilde{\boldsymbol{t}}_{k}\right\}$, are

$$
\tilde{\boldsymbol{t}}_{k}=\boldsymbol{\Omega} \boldsymbol{t}_{k}+\tau
$$

where $k=1, \ldots, K$. Since the fiducial points $\left\{\boldsymbol{Z}_{n}\right\}$ and $\left\{\boldsymbol{Y}_{n}\right\}$ in both coordinate systems are measured with some uncertainty, the corresponding transformation $\{\boldsymbol{\Omega}, \boldsymbol{\tau}\}$ will also be determined with some uncertainty. A $6 \times 6$ covariance matrix $\boldsymbol{C}_{\boldsymbol{\Theta}}$ of the six registration parameters $\boldsymbol{\Theta}=\left(\vartheta, \varphi, \rho, \tau_{x}, \tau_{y}, \tau_{z}\right)$, where the three angles $\{\vartheta, \varphi, \rho\}$ parameterize the rotation matrix $\boldsymbol{\Omega}$, can be evaluated by propagating the uncertainties of the fiducial points. Then, the covariance matrix of the transformed test point $\boldsymbol{c}\left(\tilde{\boldsymbol{t}}_{k}\right)$ can be evaluated as

$$
\boldsymbol{c}\left(\tilde{\boldsymbol{t}}_{k}\right)=\boldsymbol{J}_{\boldsymbol{t}}\left[\begin{array}{cc}
\boldsymbol{C}_{\boldsymbol{\Theta}} & 0 \\
0 & \boldsymbol{c}\left(\boldsymbol{t}_{k}\right)
\end{array}\right] \boldsymbol{J}_{\boldsymbol{t}}^{T}
$$

where the 3x9 elements of the Jacobian matrix $\boldsymbol{J}_{\boldsymbol{t}}$ are defined as the partial derivatives of the transformed test point $\tilde{\boldsymbol{t}}_{k}$ with respect to the vector $\boldsymbol{\psi}_{k}=\left[\vartheta, \varphi, \rho, \tau_{x}, \tau_{y}, \tau_{z}, x_{k}, y_{k}, z_{k}\right]$

$$
\boldsymbol{J}_{\boldsymbol{t}}=\partial \tilde{\boldsymbol{t}}_{k} / \partial \boldsymbol{\psi}_{k}
$$

Thus, the covariance matrix of the transformed test point $\boldsymbol{c}\left(\tilde{\boldsymbol{t}}_{k}\right)$ depends on the covariance matrix of the point in working frame $\boldsymbol{c}\left(\boldsymbol{t}_{k}\right)$ and on the covariance matrix $\boldsymbol{C}_{\boldsymbol{\Theta}}$ which, in turn, depends on the covariance matrices of the fiducial points $\boldsymbol{c}\left(\boldsymbol{Z}_{n}\right), \boldsymbol{c}\left(\boldsymbol{Y}_{n}\right)$. Also, $\boldsymbol{c}\left(\tilde{\boldsymbol{t}}_{k}\right)$ is affected by the placement of fiducials as the Jacobian $\boldsymbol{J}_{\boldsymbol{t}}$ is a function of the transformation parameters vector $\boldsymbol{\Theta}$ which depends on the location of fiducials. The explicit form of the matrix $\boldsymbol{C}_{\boldsymbol{\Theta}}$ is given in Eq. (A9) in the Appendix and it depends on the choice of parameterization of the rotation matrix $\boldsymbol{\Omega}$. In this study, we parameterize the axis $\boldsymbol{a}(\vartheta, \varphi)$ as follows

$$
\boldsymbol{a}(\vartheta, \varphi)=[\cos \vartheta \cos \varphi, \cos \vartheta \sin \varphi, \sin \vartheta]
$$


Once the covariance matrix of the transformed test point $\boldsymbol{c}\left(\tilde{\boldsymbol{t}}_{k}\right)$ is calculated, its eigenvalues $\left\{\lambda_{1}, \lambda_{2}, \lambda_{3}\right\}$ and eigenvectors can be determined. Then, the volume of the uncertainty ellipse of the transformed point $\tilde{\boldsymbol{t}}_{\boldsymbol{k}}$ in the reference frame is evaluated as

$$
V\left(\tilde{\boldsymbol{t}}_{k}\right)=\frac{4}{3} \pi \lambda_{1} \lambda_{2} \lambda_{3}
$$

This volume depends on the noise at test point $\boldsymbol{t}_{k}$ in the working frame, noise of the fiducials in the working and the reference frame, and on the placement of fiducials. In the same way, the volume of the uncertainty ellipse of point $\boldsymbol{t}_{k}$ measured in working frame $V\left(\boldsymbol{t}_{k}\right)$ is determined and the $k^{\text {th }}$ uncertainty expansion ratio is calculated as

$$
q_{k}=\sqrt[3]{V\left(\tilde{\boldsymbol{t}}_{k}\right) / V\left(\boldsymbol{t}_{k}\right)} .
$$

Thus, $q_{k}$ is the ratio of the radii of two spheres with equivalent volumes $V\left(\tilde{\boldsymbol{t}}_{k}\right)$ and $V\left(\boldsymbol{t}_{k}\right)$ and gives an indication of how much the uncertainty of the transformed point increases (since $q_{k} \geq 1$ ) due to the registration process. The expansion ratio is a relative measure as illustrated in Figure 3.1 and gives an indication of the precision of the registration. Note that the covariance matrix of the transformed test point $\boldsymbol{c}\left(\tilde{\boldsymbol{t}}_{k}\right)$ depends on covariance matrices of registration parameters $\boldsymbol{C}_{\boldsymbol{\theta}}$ and covariance of test point $\boldsymbol{c}\left(\boldsymbol{t}_{k}\right)$ in working frame, as given by Eq. (13). This means that two different instruments characterized by different noise levels may have similar expansion ratios. Once the expansion ratio is calculated for $k=1, \ldots, K$, a median value $Q$ is used as a characteristic for the entire working volume.

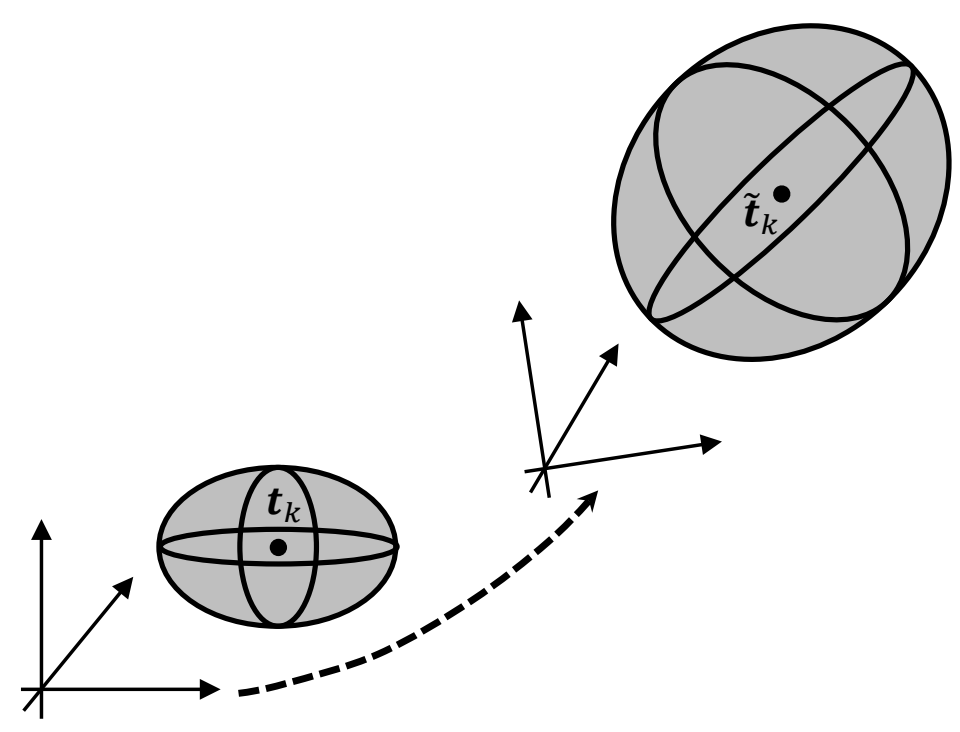

Figure 3.1. Expansion ratio $q_{k}$ as defined in Eq. (17). Test point $\boldsymbol{t}_{\boldsymbol{k}}$ from working frame is transformed to point $\tilde{\boldsymbol{t}}_{\boldsymbol{k}}$ in the reference frame by registration transformation $\{\boldsymbol{\Omega}, \boldsymbol{\tau}\}$ as in Eq. (12). A corresponding volume of the uncertainty ellipse can be derived from covariance matrix $\boldsymbol{c}\left(\boldsymbol{t}_{\boldsymbol{k}}\right)$ and $\boldsymbol{c}\left(\tilde{\boldsymbol{t}}_{\boldsymbol{k}}\right)$, respectively. 


\subsubsection{Index $W$}

Another way to gauge the performance of the registration is to check the distance between the transformed test point $\tilde{\boldsymbol{t}}_{k}$ and the corresponding test point in the reference frame, $\boldsymbol{g}_{k}$. As mentioned earlier, the distance between fiducials is commonly used as a metric rather than the distance between test points. In this study, the distances between test points are also used as a metric for registration. For each $k^{\text {th }}$ test point, the distance $d_{k}$, its variance $\operatorname{var}\left(d_{k}\right)$, and the dimensionless ratio $w_{k}$ are calculated as follows:

$$
\begin{aligned}
& d_{k}=\left\|\tilde{\boldsymbol{t}}_{k}-\boldsymbol{g}_{k}\right\| \\
& \operatorname{var}\left(d_{k}\right)=\boldsymbol{J}_{k} \boldsymbol{C}_{k} \boldsymbol{J}_{k}^{T} \\
& w_{k}=\frac{d_{k}}{\sqrt{\operatorname{var}\left(d_{k}\right)}},
\end{aligned}
$$

where the 6 x 6 covariance matrix $\boldsymbol{C}_{k}=\left[\begin{array}{cc}\boldsymbol{c}\left(\tilde{\boldsymbol{t}}_{k}\right) & 0 \\ 0 & \boldsymbol{c}\left(\boldsymbol{g}_{k}\right)\end{array}\right], \boldsymbol{c}\left(\tilde{\boldsymbol{t}}_{k}\right)$ is the covariance matrix of the transformed test point given by Eq. (13), $\boldsymbol{c}\left(\boldsymbol{g}_{k}\right)$ is a covariance of the test point measured in the reference frame, and the Jacobian vector $\boldsymbol{J}_{k}$ equals

$$
\boldsymbol{J}_{k}\left(\tilde{\boldsymbol{t}}_{k}(\tilde{x}, \tilde{y}, \tilde{z}), \boldsymbol{g}_{k}(x, y, z)\right)=\frac{1}{d_{k}}[\tilde{x}-x, \tilde{y}-y, \tilde{z}-z, x-\tilde{x}, y-\tilde{y}, z-\tilde{z}] .
$$

A value of $w_{k}<1$ indicates that the distance $d_{k}$ between transformed test point $\tilde{\boldsymbol{t}}_{k}$ and its corresponding test point $\boldsymbol{g}_{k}$ is within one standard deviation of $d_{k}$. The uncertainty of $\tilde{\boldsymbol{t}}_{k}$ and $\boldsymbol{g}_{k}$ contribute to the uncertainty of $d_{k}$ (see Eq. 18b). Similarly to the local expansion ratios $q_{k}$ and their median value $Q$, the median value $W$ for corresponding $w_{k}(k=1, \ldots, K)$ is calculated and serves as a characteristic of the entire working volume.

\subsection{4 $\boldsymbol{R M S}_{T}$}

Similar to $R M S_{F}, R M S_{T}$ (equivalent to TRE) is the RMS value of the distances between transformed test points and the corresponding test points in the reference frame and can also be calculated.

$$
R M S_{T}=\sqrt{\frac{\sum_{k=1}^{K} d_{k}^{2}}{K}}
$$

where $d_{k}$ is defined in Eq. (18a). 


\subsubsection{Proxy Index F}

Registration quality as gauged by $R M S_{F}, R M S_{T}$, $Q$, and $W$ was calculated for different combinations of $N$ fiducials. For $N=4$, the transformation $\{\Omega, \tau\}$ and the performance metrics for all possible combinations of $N$ fiducials were determined. Some of these combinations may not be reasonable, such as near-collinear or co-planar combinations, but they were processed for completeness. Out of all $M$ possible fiducials, the total number $M_{N}$ of $N$-combinations of fiducials equals $M_{N}=\frac{M !}{N !(M-N) !}$ and it increases rapidly with increasing number of fiducials $N$ used for the registration. Therefore, for $N>4$, only a subset of all possible combinations was investigated. Specifically, for $N+1$ fiducials, the first $N$ points were the same as the best $N$ fiducials which yielded the best $N$-point registration, and an additional point, $N+1$, was selected from among the remaining $(M-N)$ fiducial points one at a time and the best point was kept. This process was repeated for $N \leq 30$ and four performance metrics $Q, W, R M S_{F}$, and $R M S_{T}$ were used to select the best registration.

As mentioned, the total number of combinations $M_{N}$ increases rapidly with the number of fiducials used for registration. Also, the calculations of the expansion rates $q_{k}$ and subsequent index $Q$ are time consuming because SVD and eigenproblems have to be solved for each combination. Therefore, we propose a proxy index $F$ which is based on placement of the fiducials and instrument noise. It does not require the calculation of the registration transformation $\{\Omega, \tau\}$ but it can be used to determine which combination of fiducials may yield good registration. For $N$ fiducials, the proxy index $F$ is defined as

$$
F=\sum_{n, m}\left(\frac{\sigma_{0}\left(\boldsymbol{Z}_{n}\right)+\sigma_{0}\left(\boldsymbol{Z}_{m}\right)}{\left\|\boldsymbol{Z}_{n}-\boldsymbol{Z}_{m}\right\|}+\frac{\sigma_{0}\left(\boldsymbol{Y}_{n}\right)+\sigma_{0}\left(\boldsymbol{Y}_{m}\right)}{\left\|\boldsymbol{Y}_{n}-\boldsymbol{Y}_{m}\right\|}\right)
$$

where the summation is over all different unique pairs of indices $(n, m), n, m \leq N$, and $\sigma_{0}(\boldsymbol{Z})$ and $\sigma_{0}(\boldsymbol{Y})$ are the magnitudes of the noise (as defined in Eq. (1)) at fiducial points $\boldsymbol{Z}$ and $\boldsymbol{Y}$ in the working and reference frame, respectively.

Two conditions will result in small values of $F$ (good registration): 1) large relative distance between fiducials, and 2) small noise levels. Ideally, fiducials should be well distributed and encompass the work volume and would, thus, be placed at the periphery of the work volume (large relative distance) and at locations where the noise levels are smallest as this will result in the smallest possible $F$. However, in practical applications, this may not be possible when the noise varies within the work volume and the noise on the periphery is not the smallest. Then to obtain small values of $F$, fiducials will not be placed on the periphery or at locations where the noise is smallest but somewhere in between the two extremes.

It is assumed that small values of the proxy index $F$ should be correlated with small values of the other metrics: $Q, W, R M S_{F}$, and $R M S_{T}$. However, since no actual registration is required to 
calculate $F$, a direct one-to-one correspondence between proxy $F$ and the other metrics is not expected.

\subsubsection{Overview of metrics}

In summary, all five metrics, $Q, W, R M S_{F}, R M S_{T}$ and proxy index $F$, share one common property: a smaller numerical value corresponds to a better registration. There are, however, substantial differences between them:

- $\quad Q, W$, and $F$ are dimensionless

- $R M S_{F}, R M S_{T}$ have units of length

- $\quad Q, W, F$ and $R M S_{T}$ are based on test points while $R M S_{F}$ is based on fiducials only

- $F$ does not require registration while the remaining four can be evaluated only after registration transformation is determined. 


\section{RESULTS}

The data acquired by the three instruments, System A, B, and LT, were processed as described in the Section 3. Each dataset contains Cartesian coordinates of a measured point in the instrument's coordinate frame. The results are presented in this section.

\subsection{CHARACTERISTICS OF INSTRUMENT NOISE}

The magnitude of the noise $\sigma_{0}$ defined in Eq. (1) and determined for each fiducial point is shown in Figure 4.1. As mentioned in Section 2, the noise of the laser tracker was checked at 15 locations and the average noise, $\sigma_{0} \approx 1.7 \mu \mathrm{m}$, is almost two orders of magnitude smaller than that for Systems A and B.

A summary of the noise magnitude $\sigma_{0}$ for Systems A and B is given in Table 4.1 and Figure 4.1.

Table 4.1. Noise Characteristics $\sigma \_0$ of fiducial points for Systems A and B.

\begin{tabular}{|r|c|c|}
\hline Noise magnitude $\sigma_{0}[\mathrm{~mm}]$ & System A & System B \\
\hline Top Layer & 0.0600 & 0.1406 \\
\hline Hi Layer & 0.0556 & 0.1327 \\
\hline Mid Layer & 0.0615 & 0.1350 \\
\hline Low Layer & 0.0514 & 0.1703 \\
\hline GND Layer & 0.0661 & 0.2377 \\
\hline Average of all points & 0.0589 & 0.1633 \\
\hline Standard deviation of all points & 0.0921 & 0.0431 \\
\hline Min of all points & 0.0145 & 0.1164 \\
\hline Max of all points & 0.5782 & 0.3118 \\
\hline
\end{tabular}



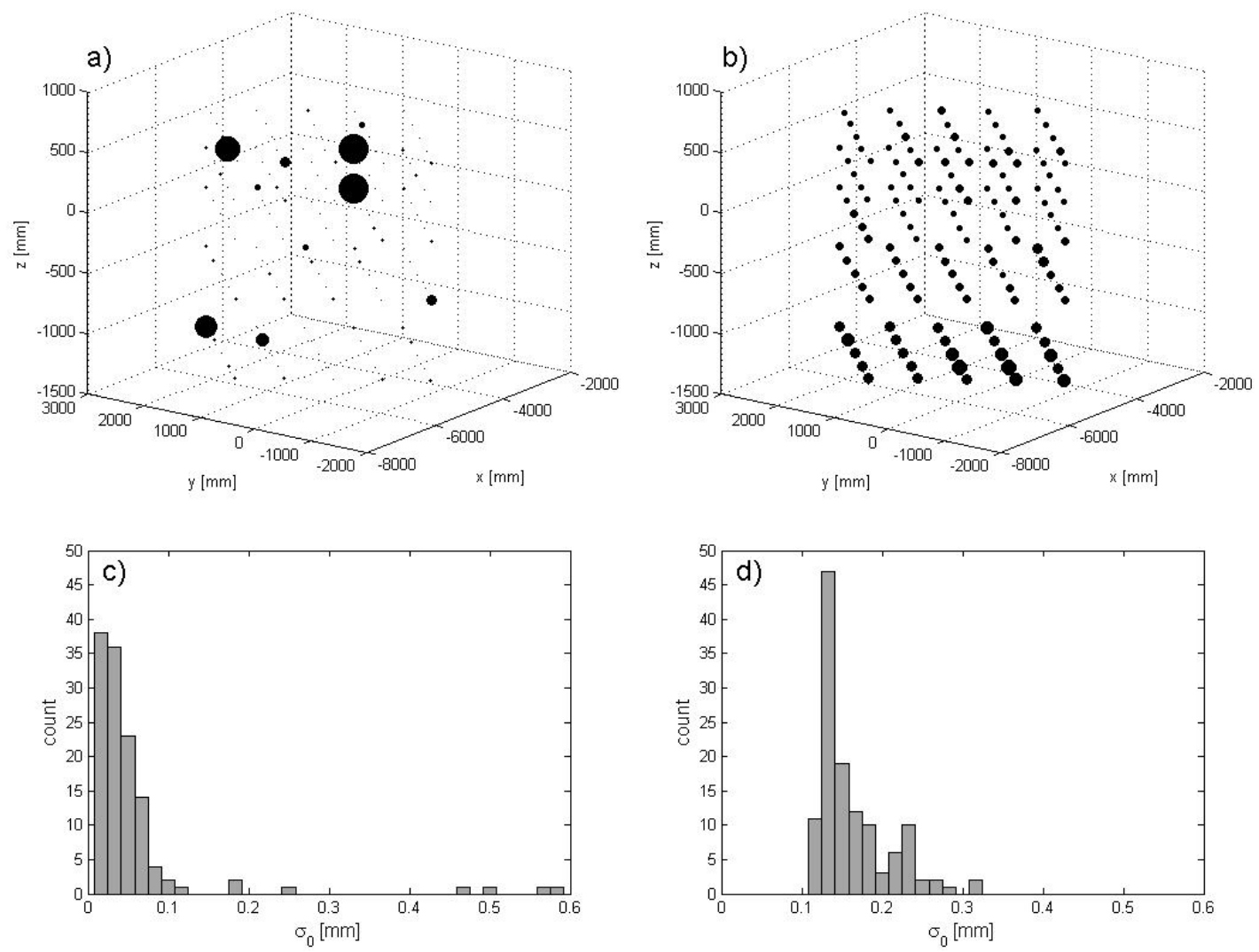

Figure 4.1. Noise magnitude $\boldsymbol{\sigma}_{\mathbf{0}}$ at fiducial points for: a) System A; b) System B. The size of the marker is proportional to $\boldsymbol{\sigma}_{\mathbf{0}}$. For better visualization and comparison, both datasets were transformed into the coordinate frame of the laser tracker. Histograms of $\boldsymbol{\sigma}_{\mathbf{0}}$ for System A and System B are shown in c) and d).

Examples of 3D point clouds from the 200 repeated measurements of the same fiducial point are shown in Figure 4.2. For each System A and B, data for two fiducials corresponding to the smallest and the largest $\sigma_{0}$ are plotted. 

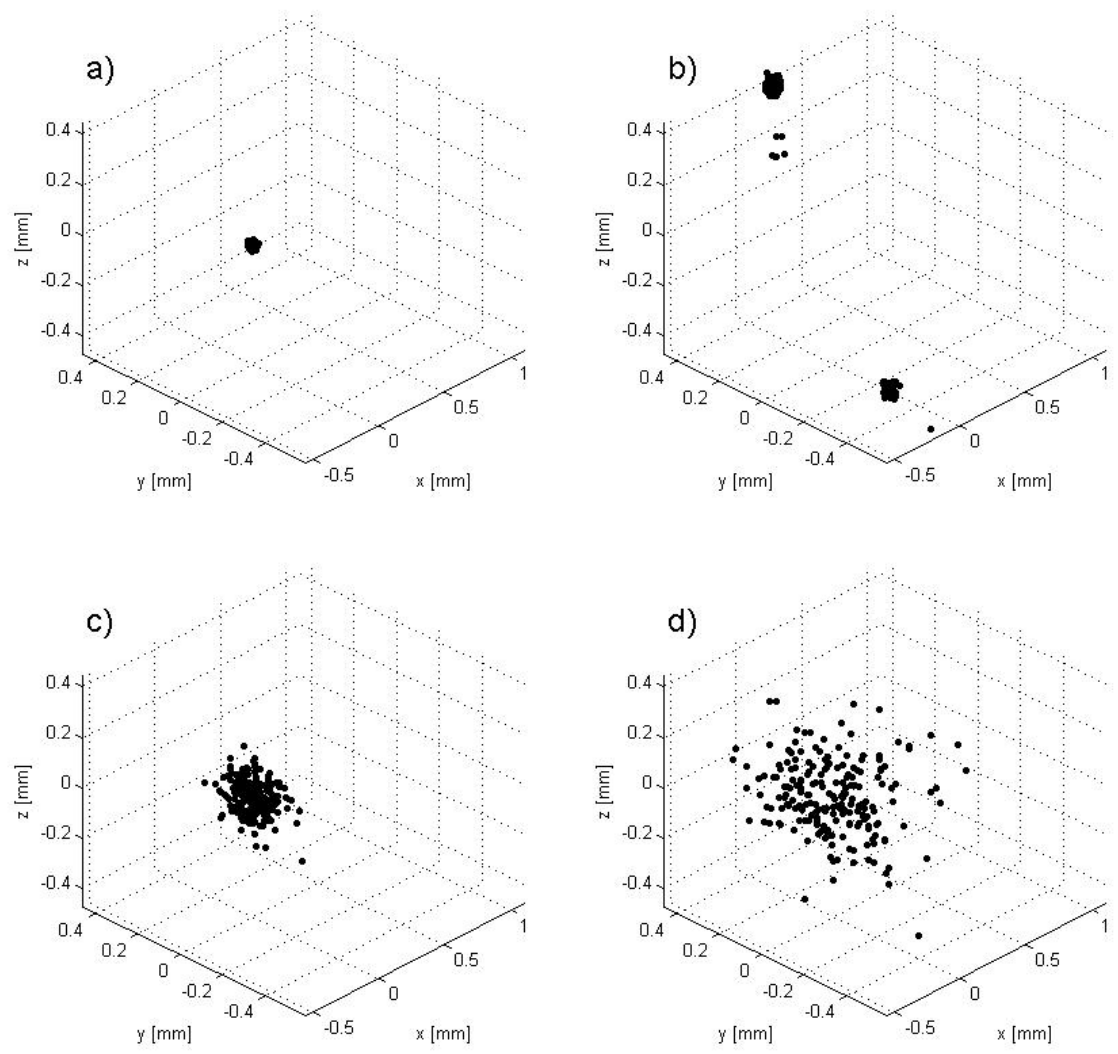

Figure 4.2. Point clouds for the 200 repeated measurement for: a) fiducial point with smallest $\boldsymbol{\sigma}_{\mathbf{0}}$ acquired by System A; b) largest $\boldsymbol{\sigma}_{\mathbf{0}}$ acquired by System A (note that the point cloud is split into two clusters); c) smallest $\boldsymbol{\sigma}_{\mathbf{0}}$ for System B; d) largest $\boldsymbol{\sigma}_{\mathbf{0}}$ for System B. For easier comparison, all datasets were transformed into the coordinate frame for the LT and the origin moved to the mean location of each dataset.

\subsection{CHECK OF RIGID BODY ASSUMPTION}

The error $L$ as defined in Eq. (2a) versus the corresponding distance $D$ measured by the laser tracker between two averaged locations of fiducials is shown in Figure 4.3. The plot contains all $(L, D)$ data calculated for all unique pairs of fiducials $M(M-1) / 2$. 


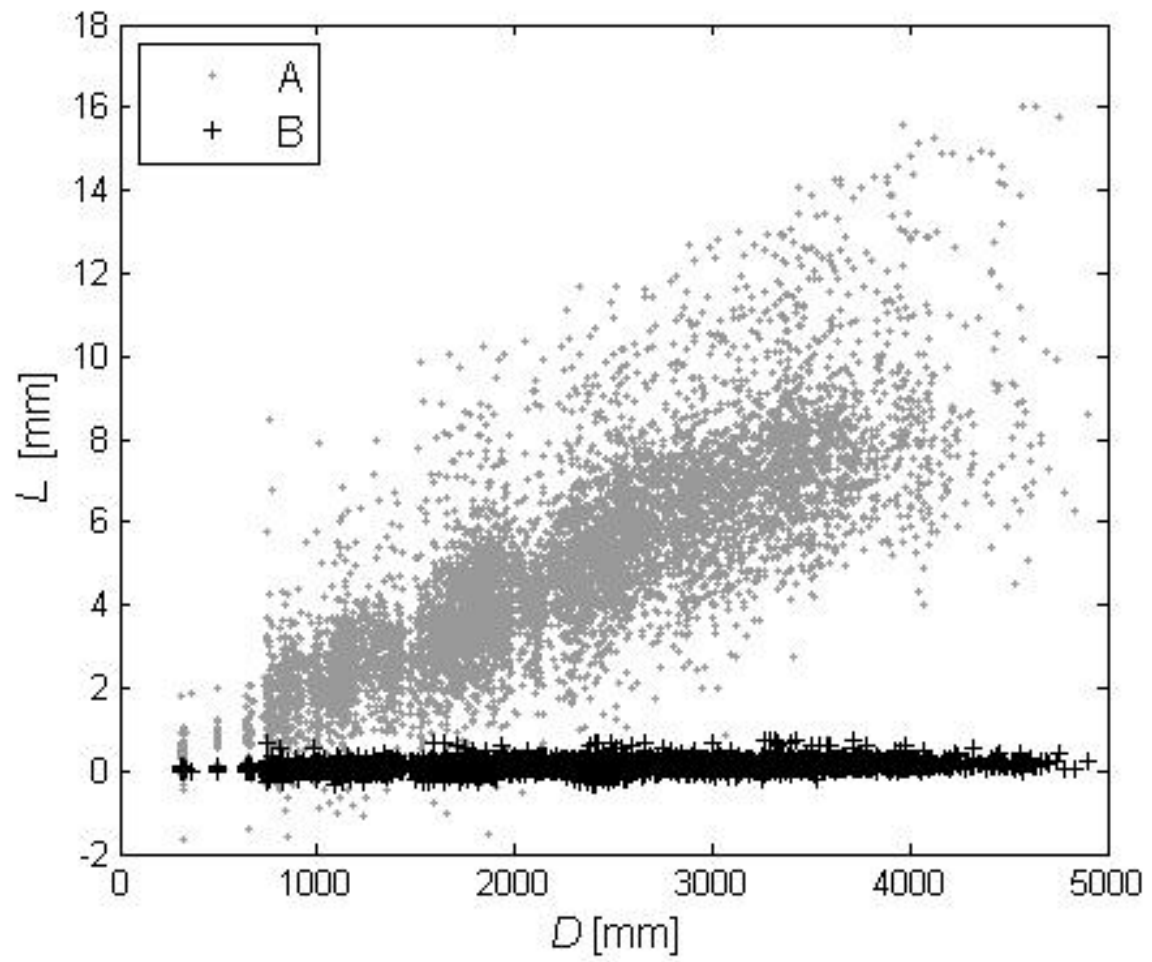

Figure 4.3. The error $L$ between the distance between two fiducials measured by System A or B and the distance $D$ between the same two fiducials as measured by laser tracker LT.

In Figure 4.4 a histogram of the dimensionless ratio $p, \frac{L}{\sqrt{\operatorname{var}(L)}}$ (Eq. 2d), is plotted. 

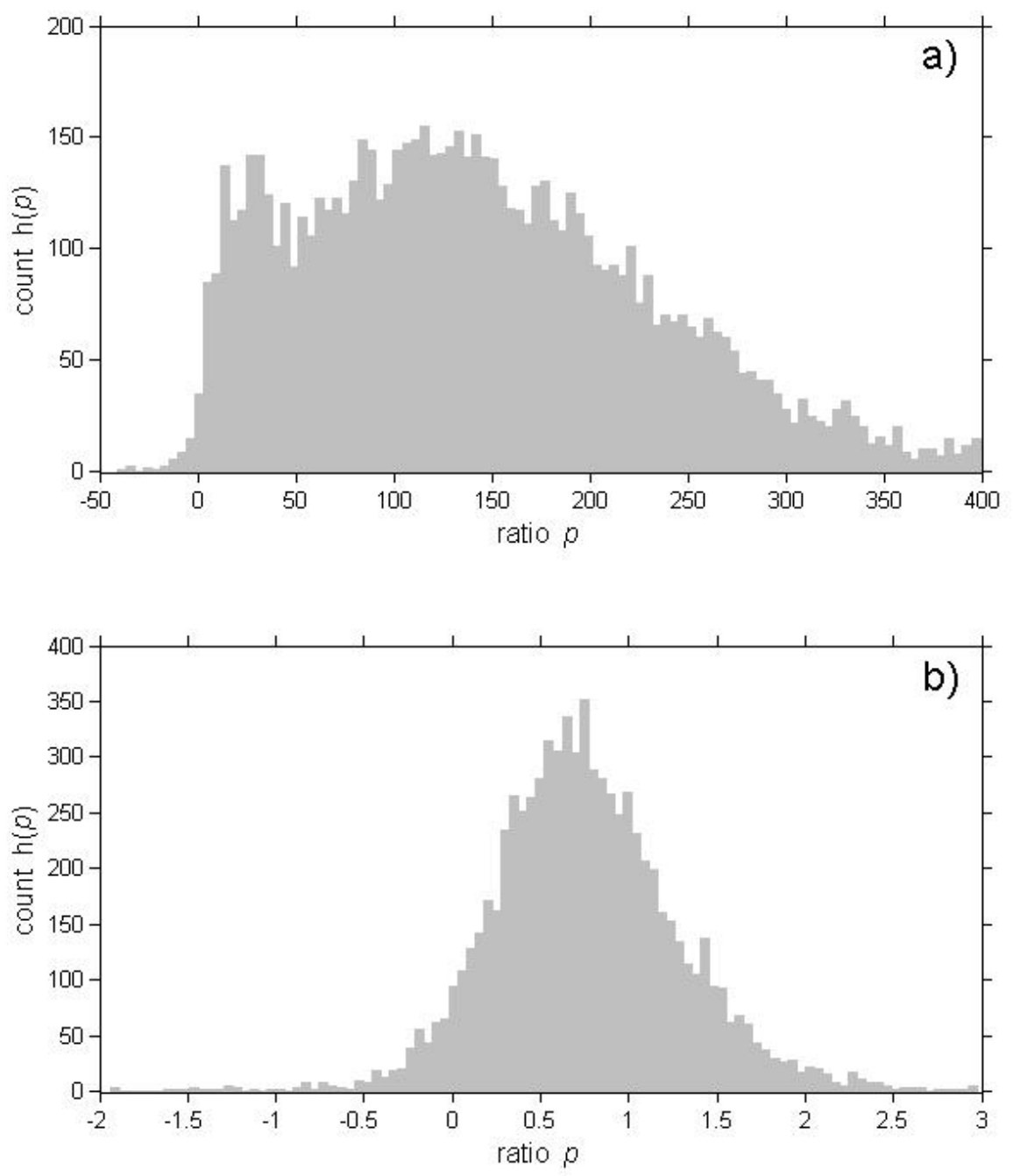

Figure 4.4. Histogram of the ratio $p, \frac{L}{\sqrt{\operatorname{var}(L)}}$, for data collected by: a) System A; b) System B.

\subsection{CALCULATIONS OF DIFFERENT REGISTRATION PERFORMANCE METRICS}

The results in this section are presented in two sections to address two different objectives: 1) find the best possible placement of a fixed number $N=4$ of fiducial points in the working volume; 2) investigate the effect of increasing $N$ on the quality of registration as gauged by different performance metrics. 


\subsubsection{Performance metrics for registration with $N=4$ fiducials}

Out of a total of $M$ fiducials, all $M_{N}$ possible combinations of $N=4$ fiducials were processed. For each combination, the registration transformation $\{\boldsymbol{\Omega}, \boldsymbol{\tau}\}$ was determined and indices $F, Q$, and $W$ as well as $R M S_{F}$ and $R M S_{T}$ were calculated. In Figure 4.5, histograms of $F$ and $Q$ are plotted for the registration of System A to LT, System B to LT, and System A to System B.
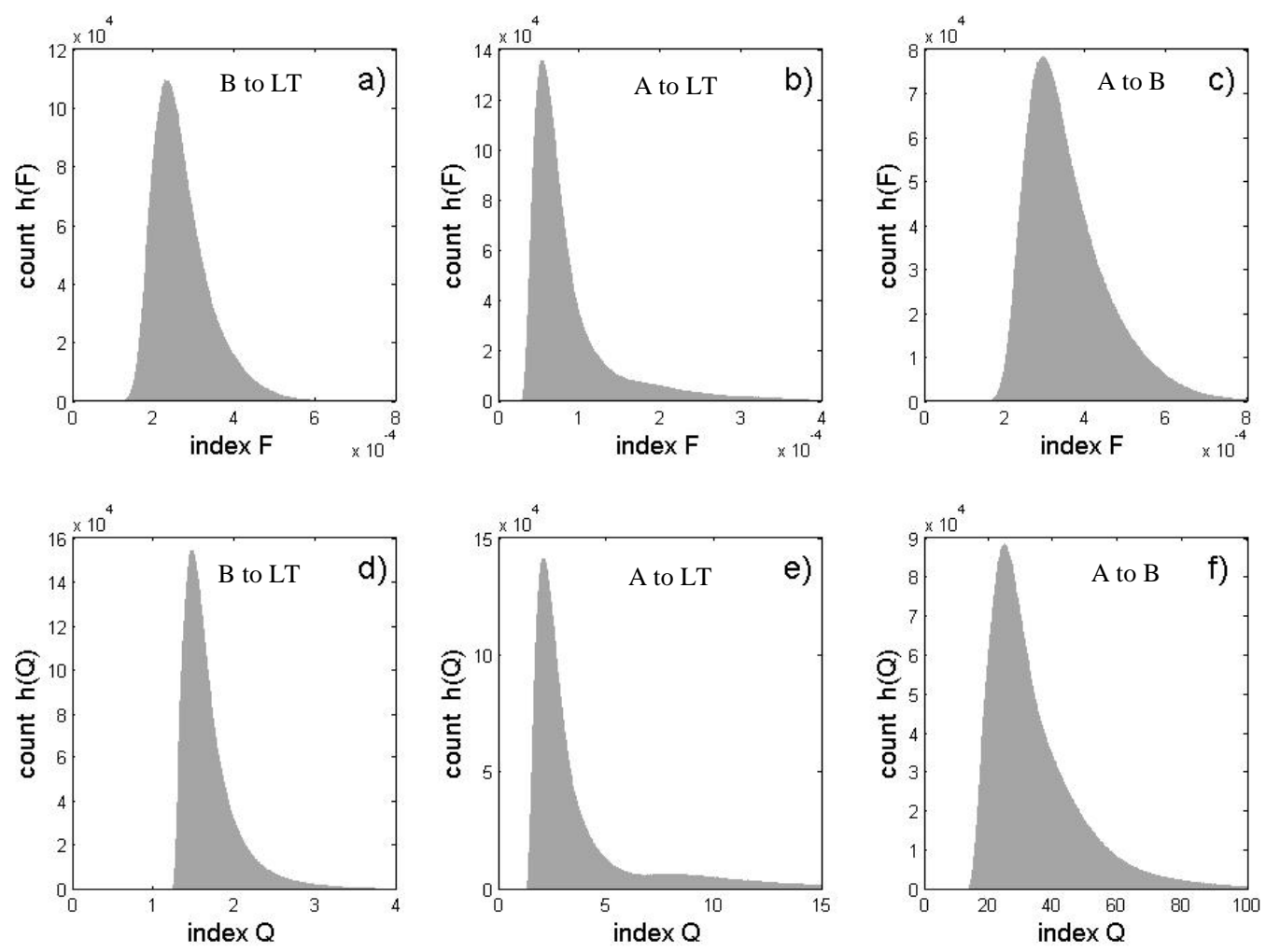

Figure 4.5. Distribution of indices $F$ and $Q$ for all possible combinations of four fiducials $N=4$ selected from $M=125$ fiducials $\left(M_{N}=9691375\right.$ ). a-c) index $F$ for (B to LT), (A to LT), and (A to B) registration, respectively; d-f) index $Q$ for (B to LT), (A to LT), (A to B) registration, respectively.

Index $Q$ is the median of the expansion ratios $q_{k}$ evaluated at test points and it characterizes the whole work volume. Individual $q_{k}$ depends on both the registration quality and the location of $k^{\text {th }}$ test point, as shown in Figure 4.6. Index $Q$ was sorted in ascending order so that rank $k_{Q}=1$ corresponds to the best registration (smallest $Q$ ) and $k_{Q}=M_{N}$ corresponds to the worst registration (largest $Q$ ). Figure 4.6a shows the individual $q_{k}$ for an "average" registration $\left(k_{Q}=\right.$ $0.4 M_{N}$ ) while Figure $4.6 \mathrm{~b}$ shows the individual $q_{k}$ for the best registration.

Figure 4.7 shows the histograms of $R M S_{T}, R M S_{F}$, and their ratio $s=R M S_{T} / R M S_{F}$ for (B to LT) and (A to LT) registrations. Histograms for (A to B) registration are almost identical with 
corresponding histograms for (A to LT) registration and are not shown. Data presented in this figure were obtained with method 1 (solid line) and method 2 (dotted line). Recall that method 1 uses the average of 200 repeat measurements while method 2 uses each of the repeated measurement.
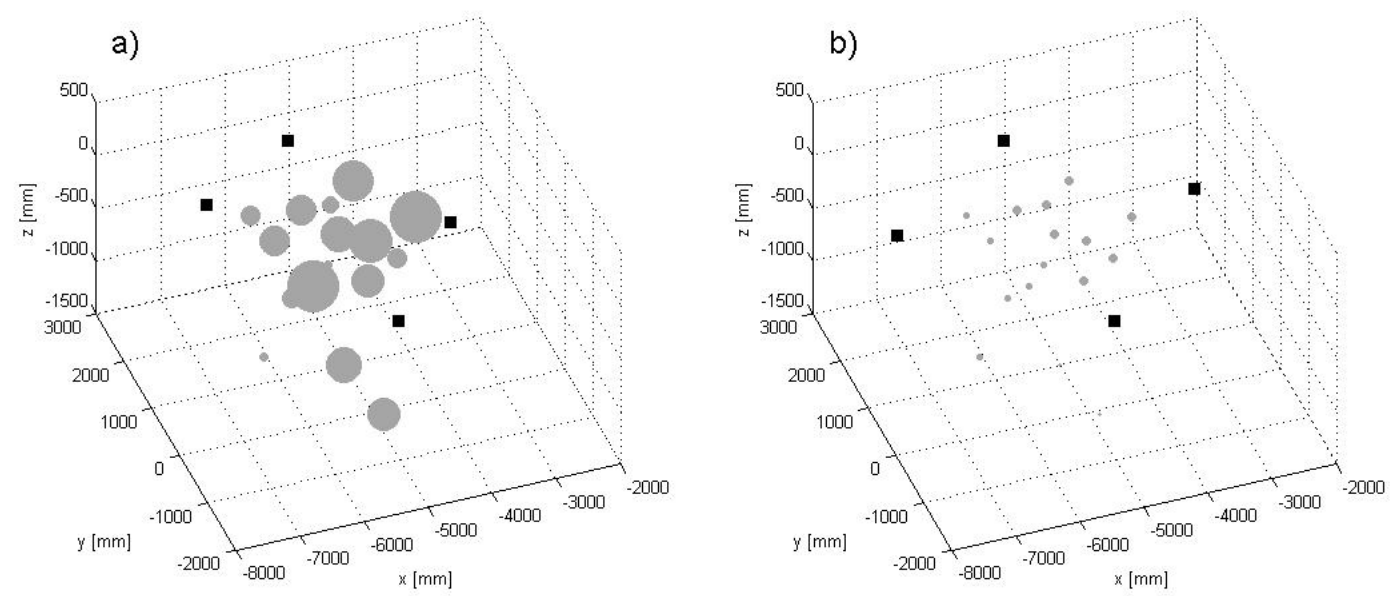

Figure 4.6. Example of individual indices $q_{k}(k=1, \ldots, 16)$ for the 16 test points. The size of each gray circle is proportional to the value of the corresponding $q_{k}$. The four black squares mark the locations of the four fiducials used for registration: a) System A to B; b) System B to LT. Placement of fiducials corresponds to: a) rank $k_{Q}=0.4 M_{N}$ of sorted in ascending order index $Q$; b) rank $k_{Q}=1$ (the smallest $Q$ ). Both graphs are in LT coordinate frame. 

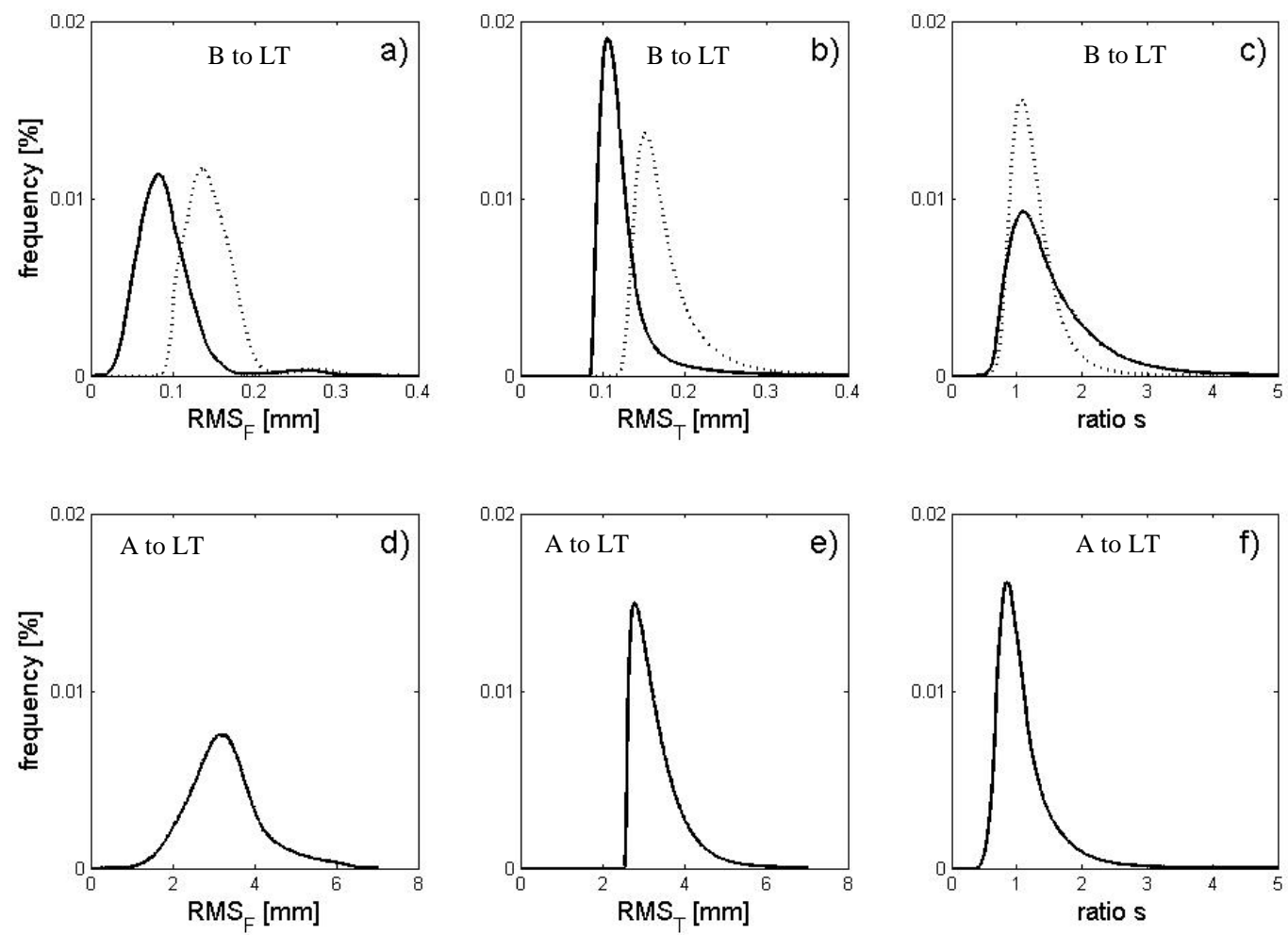

Figure 4.7. Histograms of RMS for registrations based on all possible combinations of $N=4$ fiducials; solid line: RMS calculated using method 1, dotted line: using method 2. There is only a slight difference between the solid and dotted lines in plots $(\mathrm{d}-\mathrm{f})$ and this difference is not visible in the plots. a) $R M S_{F}$ of fiducials for (B to LT) registration; b) $R M S_{T}$ of test points for (B to LT) registration; c) ratio s of $R M S_{T}$ to $R M S_{F}$ for (B to LT) registration; d) $R M S_{F}$ of fiducials for (A to LT) registration; e) $R M S_{T}$ of test points for (A to LT) registration; f) ratio s of $R M S_{T}$ to $R M S_{F}$ for (A to LT) registration.

The correlation coefficients between $R M S_{T}$ and $R M S_{F}$ for System A to LT, System B to LT, and System A to B are $-0.0011,0.3832$, and 0.0059 , respectively.

Figure 4.8 shows the histograms of index $W$. 

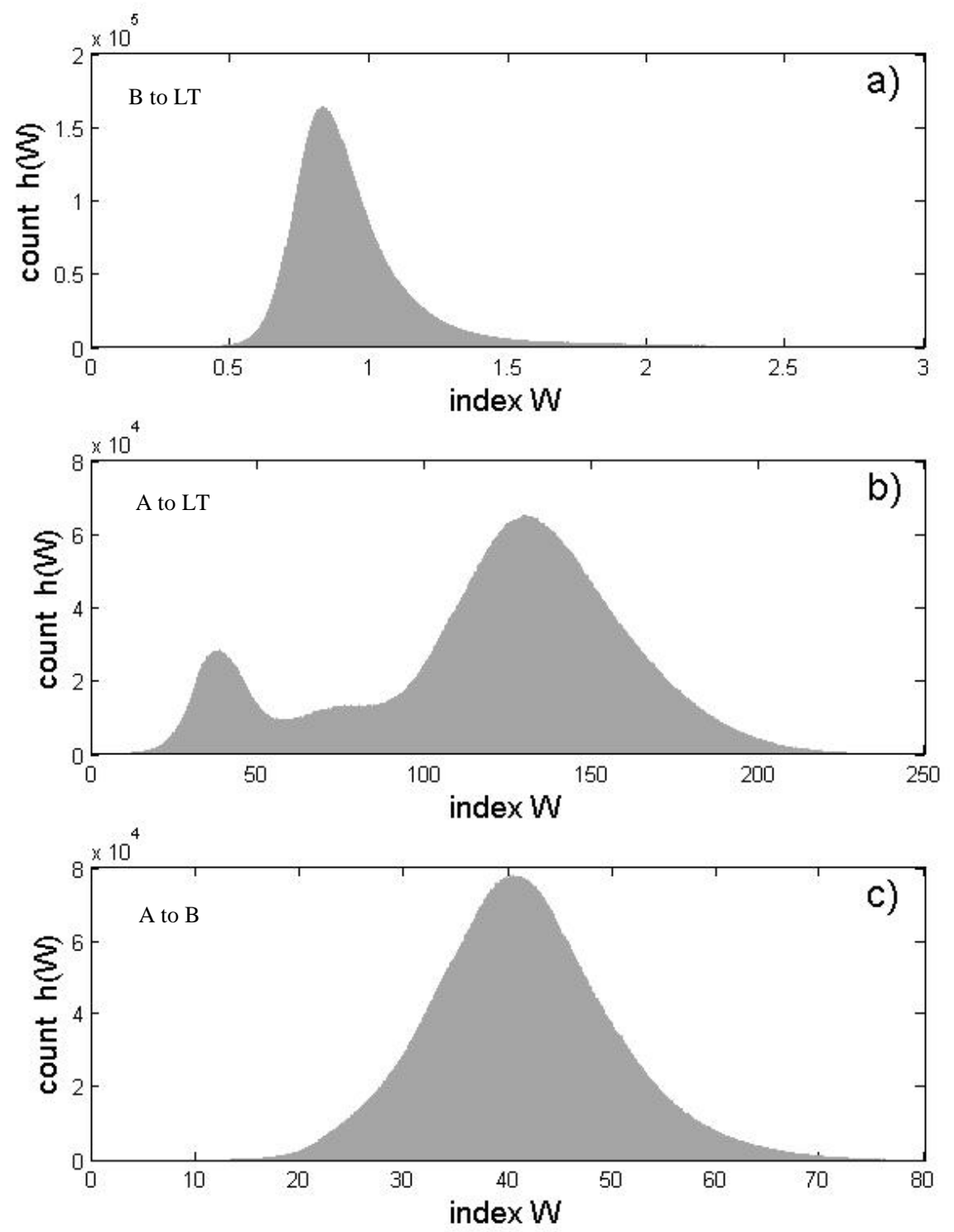

Figure 4.8. Distribution of index $W$ for registering: a) B to LT; b) A to LT; c) A to B.

Plots in Figure 4.9 - Figure 4.11 (registration of System B to LT, System A to LT, and System A to $\mathrm{B}$, respectively) show the comparisons between the proxy index $F$ and the other four metrics. All possible $M_{N}$ combinations of $N=4$ fiducials were processed and for each metric, the $m^{\text {th }}$ combination yielding the smallest value of the metric was determined. For example, $m\left(R M S_{F}\right)$ indicates the combination of fiducials resulting in the smallest $R M S_{F}$. In each of the subplots (a $-\mathrm{e})$, a given metric is plotted for the five combinations $m(F), m(Q), m(W), m\left(R M S_{F}\right)$, and $m\left(R M S_{T}\right)$. 

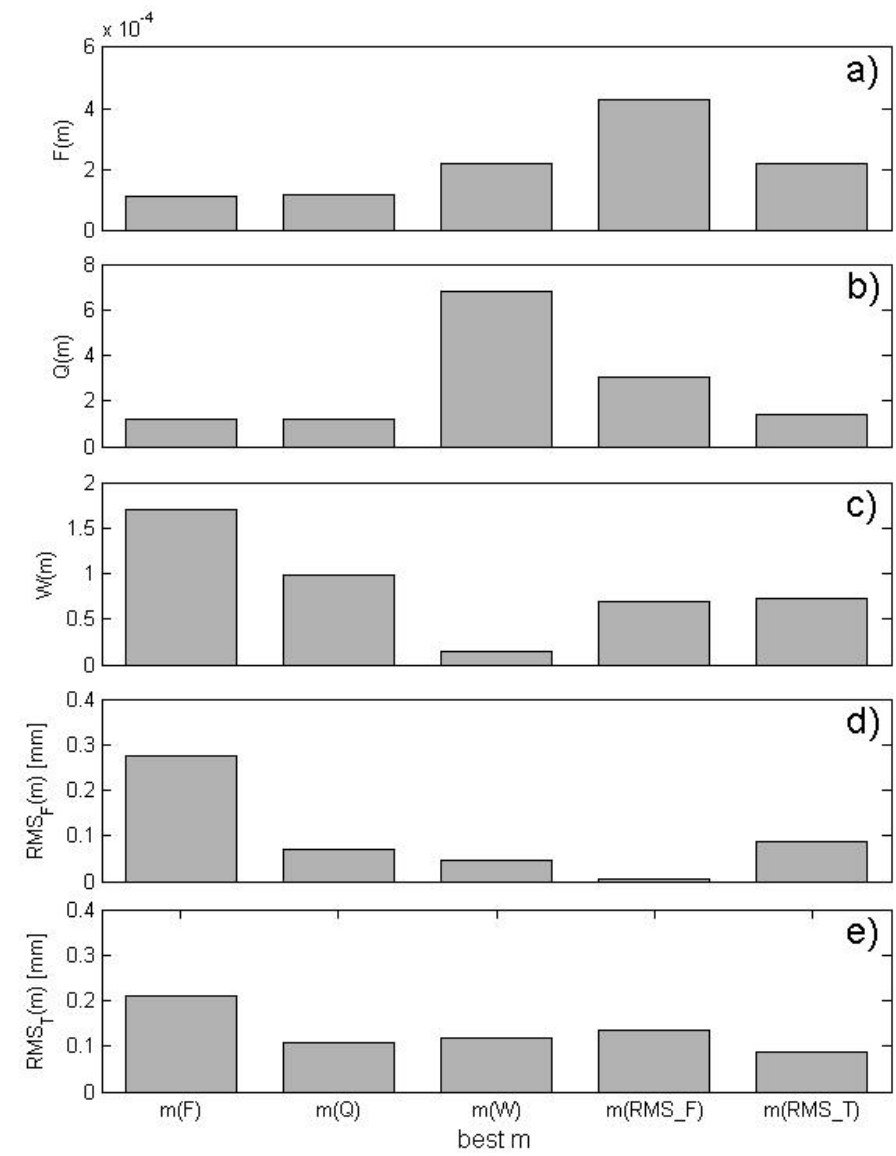

Figure 4.9. Relationship of the proxy index $F$ and four performance metrics: $Q, W, R M S_{F}$, and $R M S_{T}$ for System B registered to LT using $N=4$ fiducials. Individual plots: a) proxy index $F$ for the five different combinations of fiducials yielding the smallest $F, Q, W, R M S_{F}$ and $R M S_{T}$; b) index $Q$ for the same five combinations; similarly for c) index $W$; d) $R M S_{F}$; e) $R M S_{T}$. 

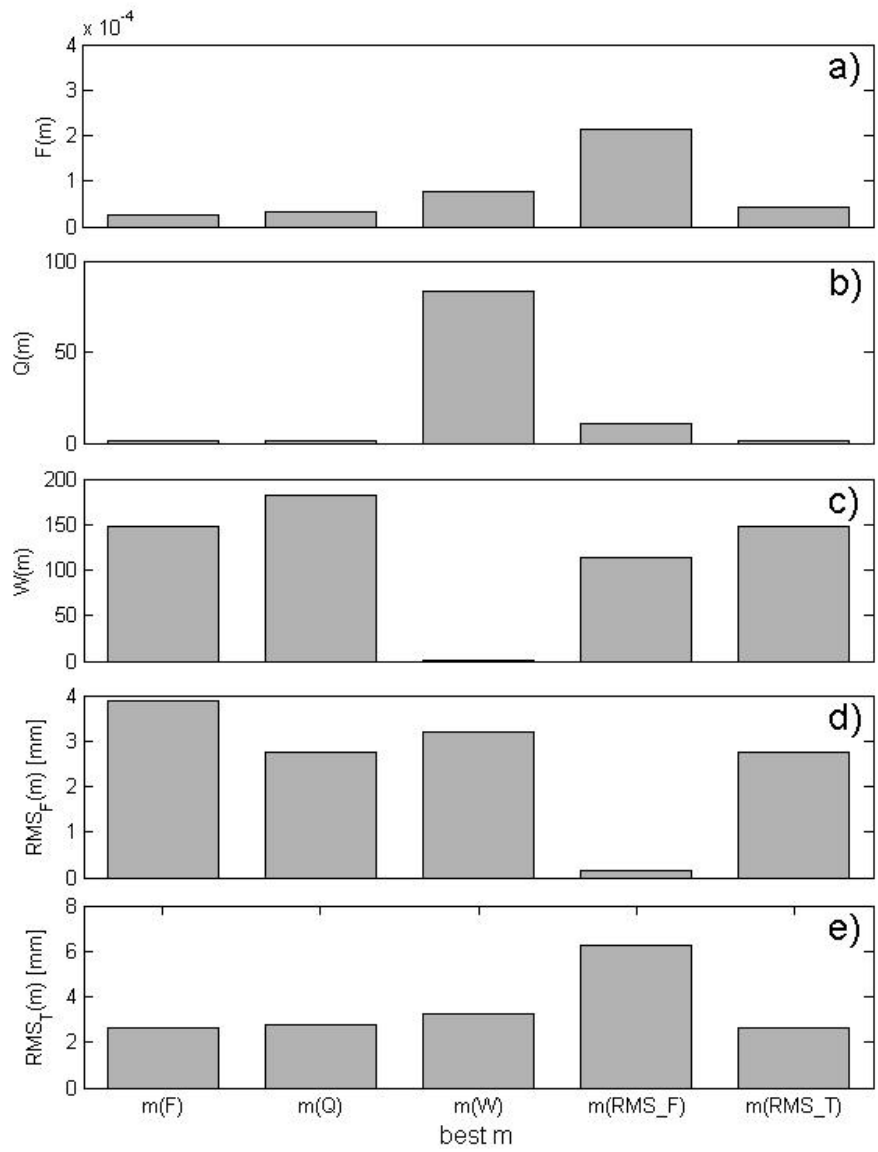

Figure 4.10. Relationship of proxy index $F$ and four performance metrics: $Q, W, R M S_{F}$, and $R M S_{T}$ for System A registered to LT using $N=4$ fiducials. Individual plots: a) proxy index $F$ for the five different combinations of fiducials yielding the smallest $F, Q, W, R M S_{F}$ and $R M S_{T}$; b) index $Q$ for the same five combinations; similarly for c) index $W$; d) $R M S_{F}$; e) $R M S_{T}$. 

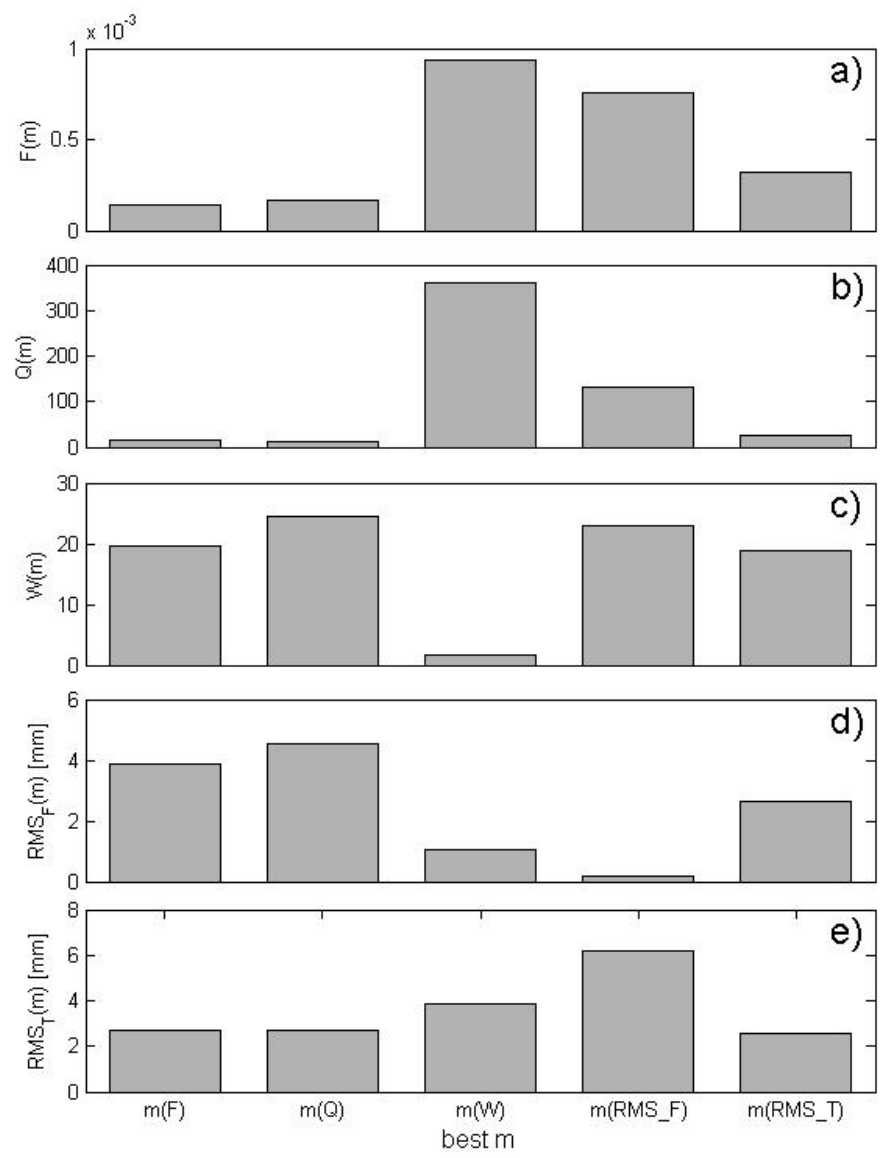

Figure 4.11. Relationship of proxy index $F$ and four performance metrics: $Q, W, R M S_{F}$, and $R M S_{T}$ for System A registered to System B using $N=4$ fiducials. Individual plots: a) proxy index $F$ for the five different combinations of fiducials yielding the smallest $F, Q, W, R M S_{F}$, and $R M S_{T}$; b) index $Q$ for the same five combinations; similarly for c) index $W$; d) $R M S_{F}$; e) $R M S_{T}$.

Figure 4.12 compares the proxy index $F$ and index $Q$ for $N=4$ fiducials. In each of the subplots $(\mathrm{a}-\mathrm{c}$ ), the ten smallest values of $Q$ are displayed as black bars and the ten values of $Q$ (light gray bars) corresponding to the ten combinations of fiducials yielding the ten smallest proxy index $F$ are plotted. The bottom horizontal axis shows the rank $k_{Q}$ for the black bars and the top horizontal axis shows the rank $k_{Q}$ for the gray bars. 

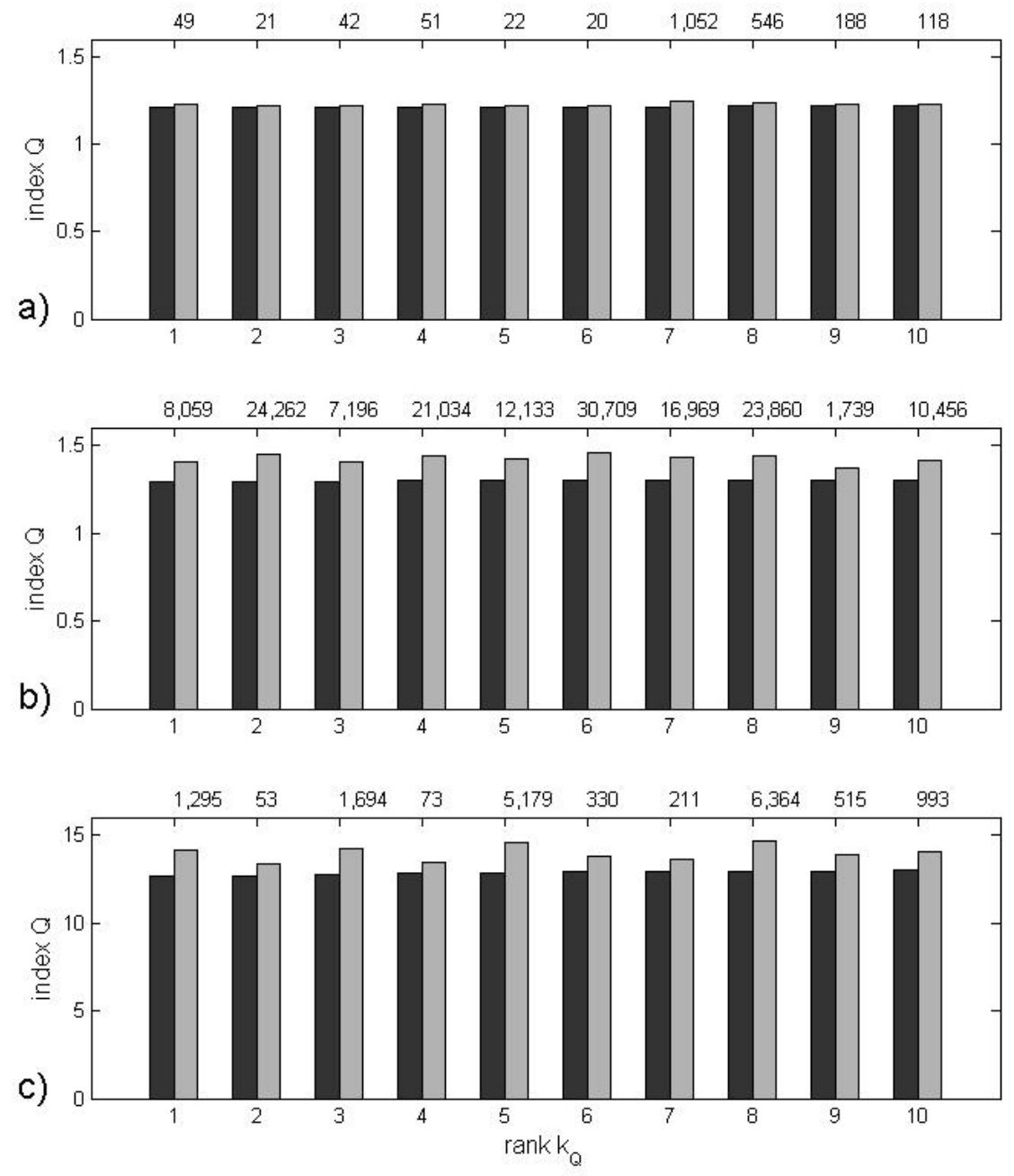

Figure 4.12. First ten values of index $Q$ sorted in ascending order (dark bars) and ten values of $Q$ for ranks $k_{Q}$ corresponding to the ten smallest values of index $F$ (light gray bars). Numbers on upper horizontal axes of each subplot are ranks $k_{Q}$ corresponding to ten smallest values of index $F$. Results for registration: a) B to LT; b) A to LT; c) A to B.

The influence of the noise in the selection of $N=4$ fiducials is shown in Figure 4.13. Plots in Figure 4.13(a, c) show the locations of the selected fiducials for variable noise, $\sigma_{0}$, and Figures 4.13(b, d) show the locations of the selected fiducials for constant noise where the constant noise is the average $\sigma_{\text {avg }}$ (averaged over all $\sigma_{0}$ ). For the four combinations of fiducials shown in Figure 4.13(a - d), the corresponding values of index $Q(\mathrm{a}-\mathrm{d})=[1.35,1.74,1.33,1.64]$. 

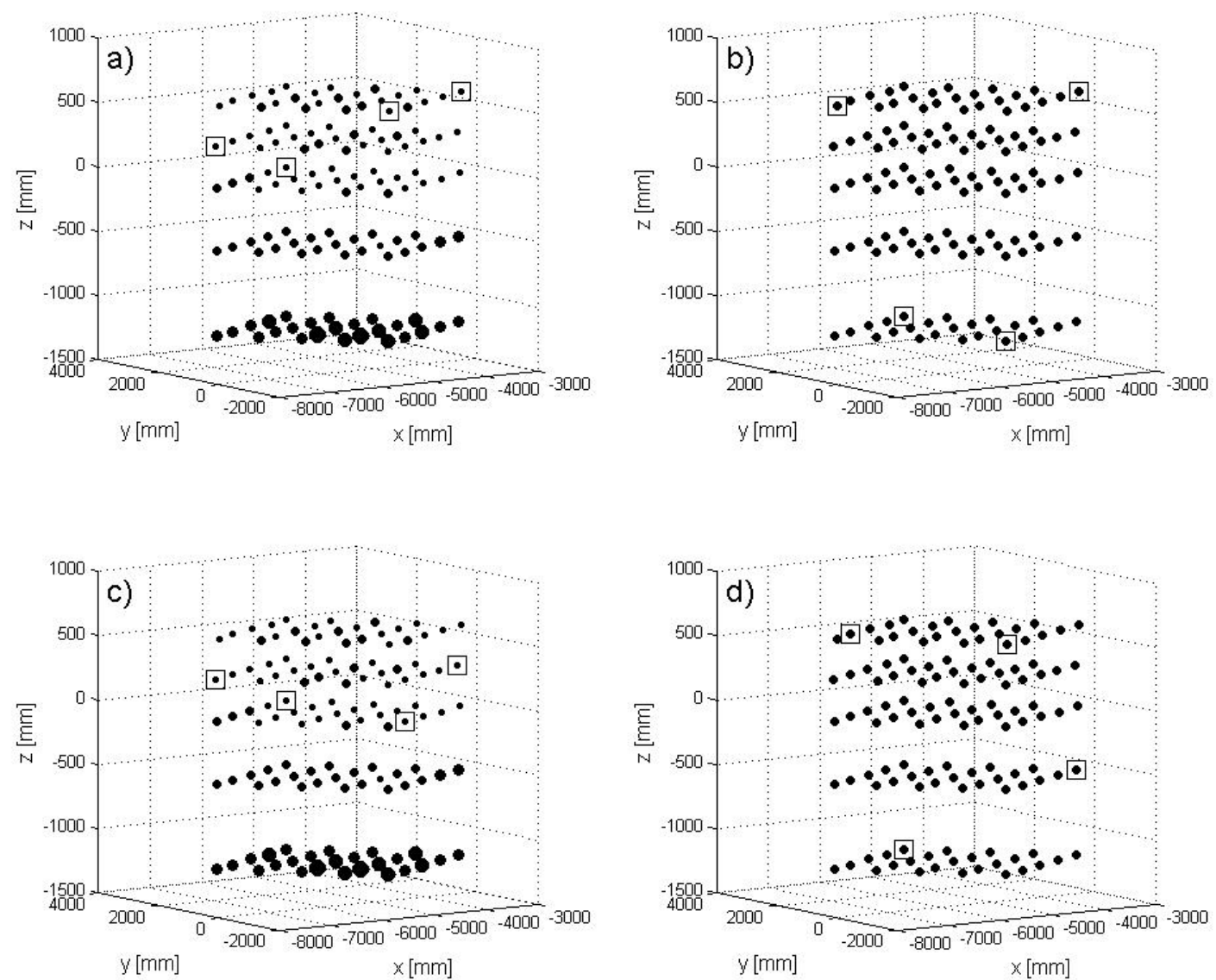

Figure 4.13. Influence of variable noise on the choice of fiducials location, registration of System B to LT: a) based on the best proxy index $F$ and actual variable noise $\boldsymbol{\sigma}_{\mathbf{0}}$, corresponding value of $Q=1.35$; b) based on the best proxy index $F$ and constant average noise $\boldsymbol{\sigma}_{\boldsymbol{a v g}}, Q=1.74$; c) based on the best index $Q$ and actual variable noise $\boldsymbol{\sigma}_{\mathbf{0}}, Q=1.33$; d) based on the best index $Q$ and constant average noise $\boldsymbol{\sigma}_{\boldsymbol{a v g}}$, $Q=1.64$. Size of the circular markers is proportional to the magnitude of the noise; locations of selected fiducials are marked by squares.

\subsubsection{Effect of the number of fiducials on registration performance}

To determine the effect of the number of fiducials on the performance of the registration, a slightly different approach had to be applied than that used in Section 4.3.1. For all possible combinations $M_{N}$ for $N>4$ fiducials, the approach in Section 4.3.1 is not tenable as the number of combinations increases rapidly with increasing $N$ (for $N=5$ fiducials, $M_{N}=234531275$ ).

Therefore, the best possible combination of $N=4$ fiducials corresponding to the smallest index $Q$ was selected as a starting point. Then, the best combination (based on index $Q$ ) of $N+1$ 
fiducials was identified as described at the end of Section 3.3. Figure 4.14 shows the value of index $Q$ calculated for four cases: 1) combination of $N=4$ fiducials corresponding to rank $k_{Q}=1$ (smallest value of $Q$ ); 2) $N=5$, four fiducials used in case 1 and the $5^{\text {th }}$ fiducial is selected from the remaining 121 to yield the smallest $Q$; 3) combination of $N=4$ fiducials corresponding to rank $k_{Q}=M_{N} / 2$ (middle of the sorted list of $Q$, i.e., "average" registration performance based on the $Q$ index); 4) $N=5$, four fiducials used in case 3 and the $5^{\text {th }}$ fiducial is selected from the remaining 121 to yield the smallest $Q$.
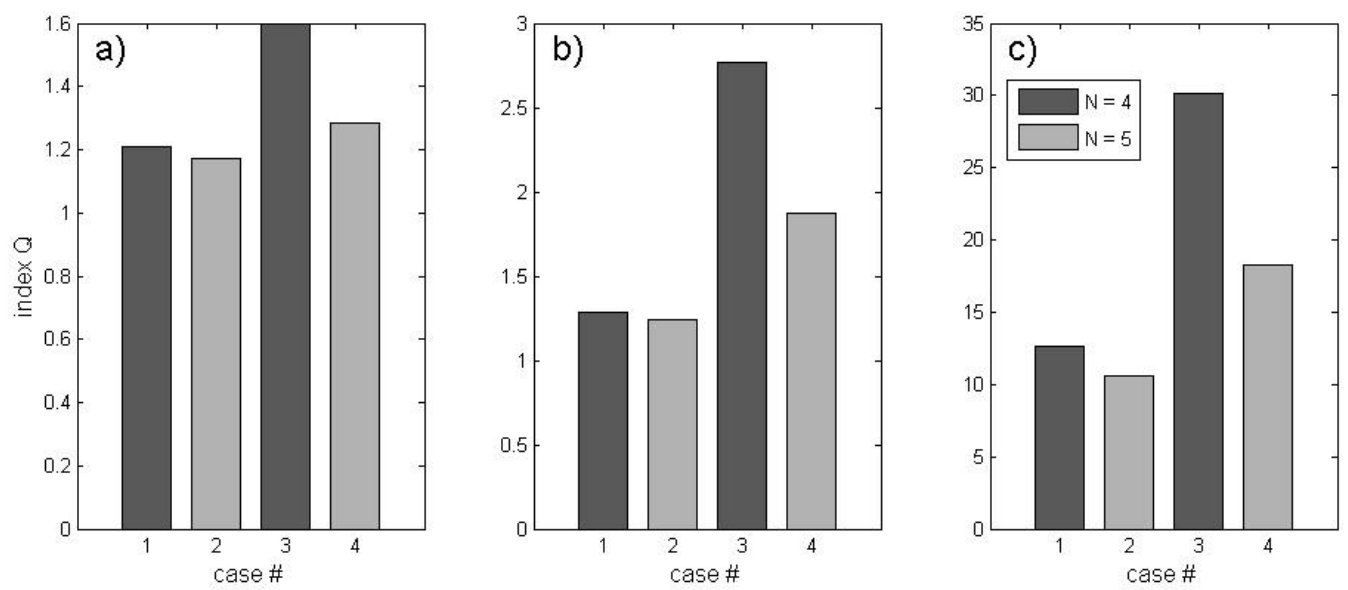

Figure 4.14. Comparison of registration performance for $N=4$ and $N=5$ fiducials used to register: a) System B to LT; b) System A to LT; c) System A to B. Registration performance based on index $Q$ where lower $Q$ indicates better registration.

Similarly, the best possible combination of $N=4$ fiducials corresponding to the smallest value of proxy index $F$ was selected. Then, the best combination of $N+1$ fiducials was identified based on proxy index $F$. Figure 4.15 shows the value of index $Q$ calculated for five cases: 1) combination of $N=4$ fiducials corresponding to rank $k_{F}=1$ (smallest value of $F$ ); 2) $N=5$, use the four fiducials from case 1 and the $5^{\text {th }}$ fiducial is selected from the remaining 121 fiducials to yield the smallest $F$; 3) similar to case 2 but instead of taking only the best combination of $N=4$ fiducials, the first $10^{6}$ combinations $\left(k_{F} \leq 10^{6}\right)$ were checked and for each combination, the fifth fiducial from the remaining 121 fiducials that yielded the smallest $F$ was selected - then, from the resulting $10^{6}$ combinations of $N=5$ fiducials, the combination with the smallest $F$ was selected. The search strategy applied in case 2 and 3 cannot guarantee that the smallest proxy $F$ is found, but in case 3, a much larger number of combinations $\left(10^{6}\right)$ is checked; 4) combination of $N=4$ fiducials corresponding to rank $k_{F}=M_{N} / 2$ (middle of the sorted list of $F$ ); 5) $N=5$, four fiducials used in case 4 and the $5^{\text {th }}$ fiducial is selected from the remaining $(M-N)$ fiducials to yield the smallest $F$. 

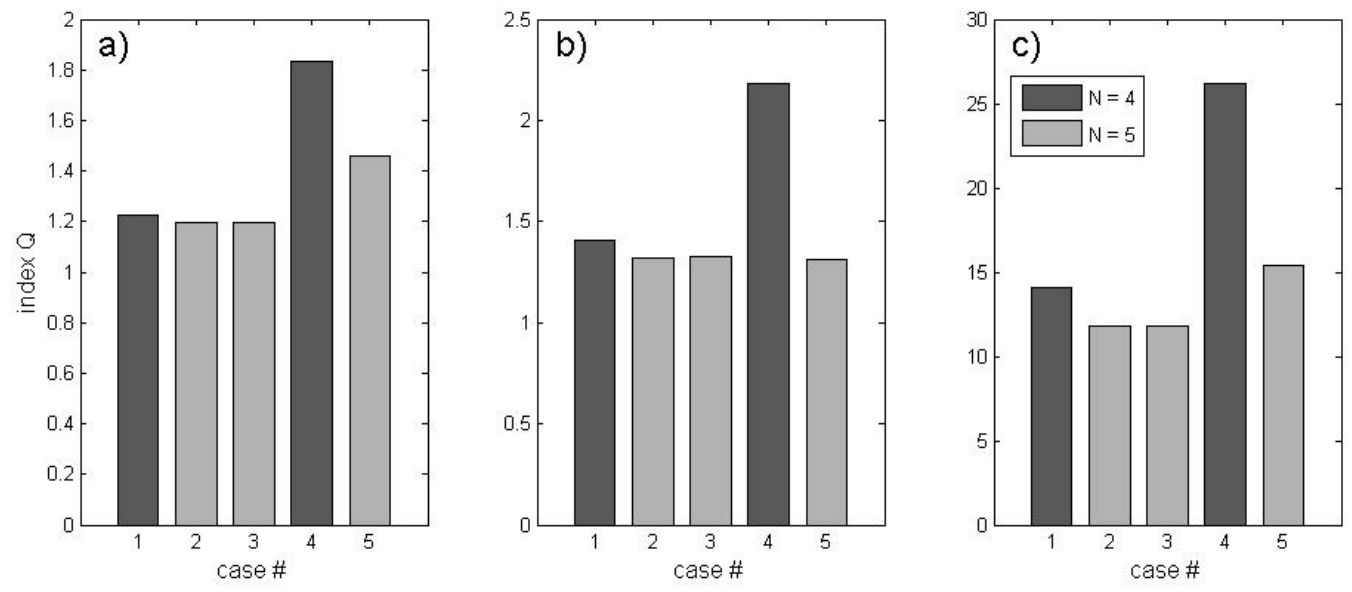

Figure 4.15. Comparison of registration performance for $N=4$ and $N=5$ fiducials used to register: a) System B to LT; b) System A to LT; c) System A to B. Registration performance based on proxy index F where lower $F$ indicates better registration.

In Figure 4.16, two values of index $Q$ obtained from two different selection strategies versus the number of fiducials $N$ used in registration are plotted. For $N=4$, the smallest possible $Q$ is plotted with a (+) symbol, while the $\left(^{*}\right)$ symbol corresponds to rank $k_{Q}=M_{N} / 2$ (middle of sorted $Q$ list). For consecutive number of fiducials, the $N+1$ fiducial is selected from the remaining $M-N$ fiducials so that the resulting $Q$ is the smallest (+) or is in the middle of sorted $Q$ list with rank $k_{Q}=(M-N) / 2(*)$. A similar procedure was repeated for $R M S_{F}$ and $R M S_{T}$ and the resulting dependence on the number of fiducials $N$ is shown in Figure 4.17 and Figure 4.18. 

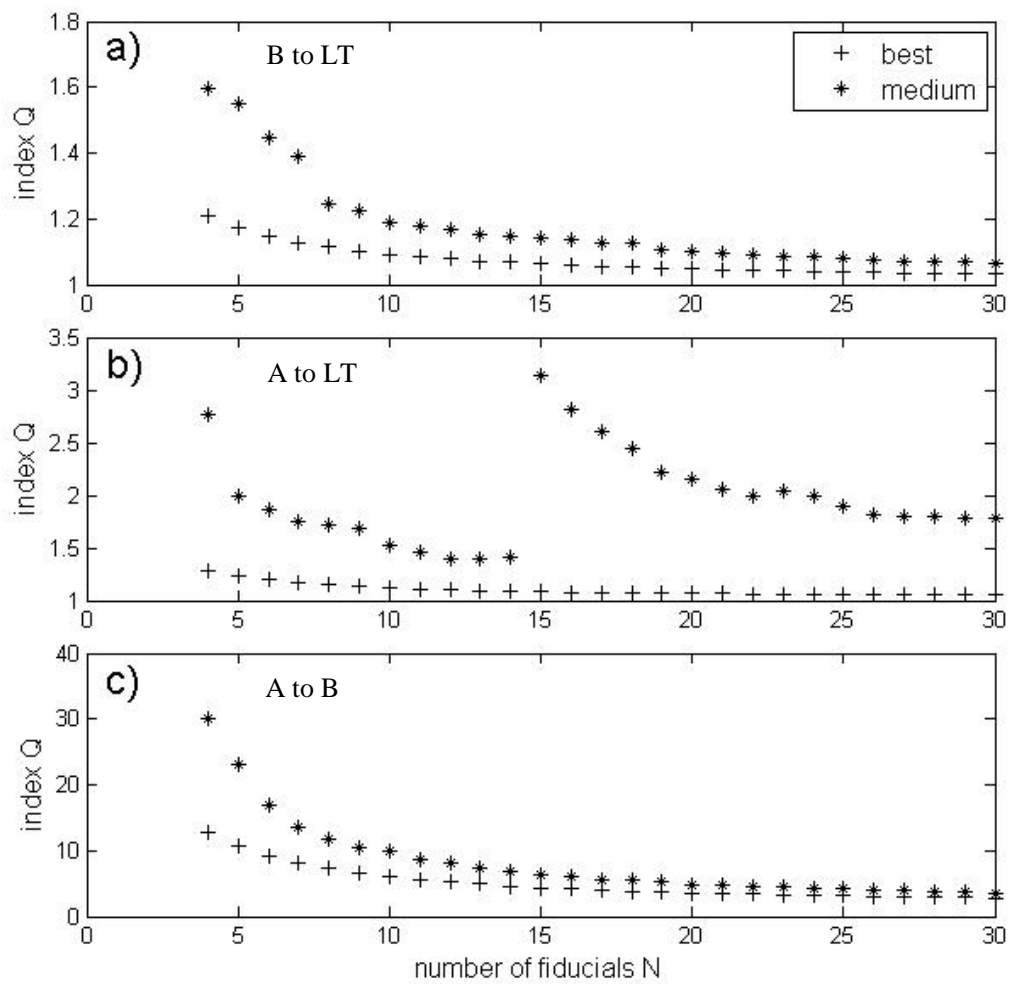

Figure 4.16. Index $Q$ versus the number of fiducials $N$ used in the registration. Two criteria for selecting the best registration were used for each combination of $N$ : based on the smallest $Q(+)$ and medium $Q\left({ }^{*}\right)$ from the middle of a sorted $Q$ list. Individual plots are for registration of: a) System B to LT; b) System A to LT; c) System A to B. 

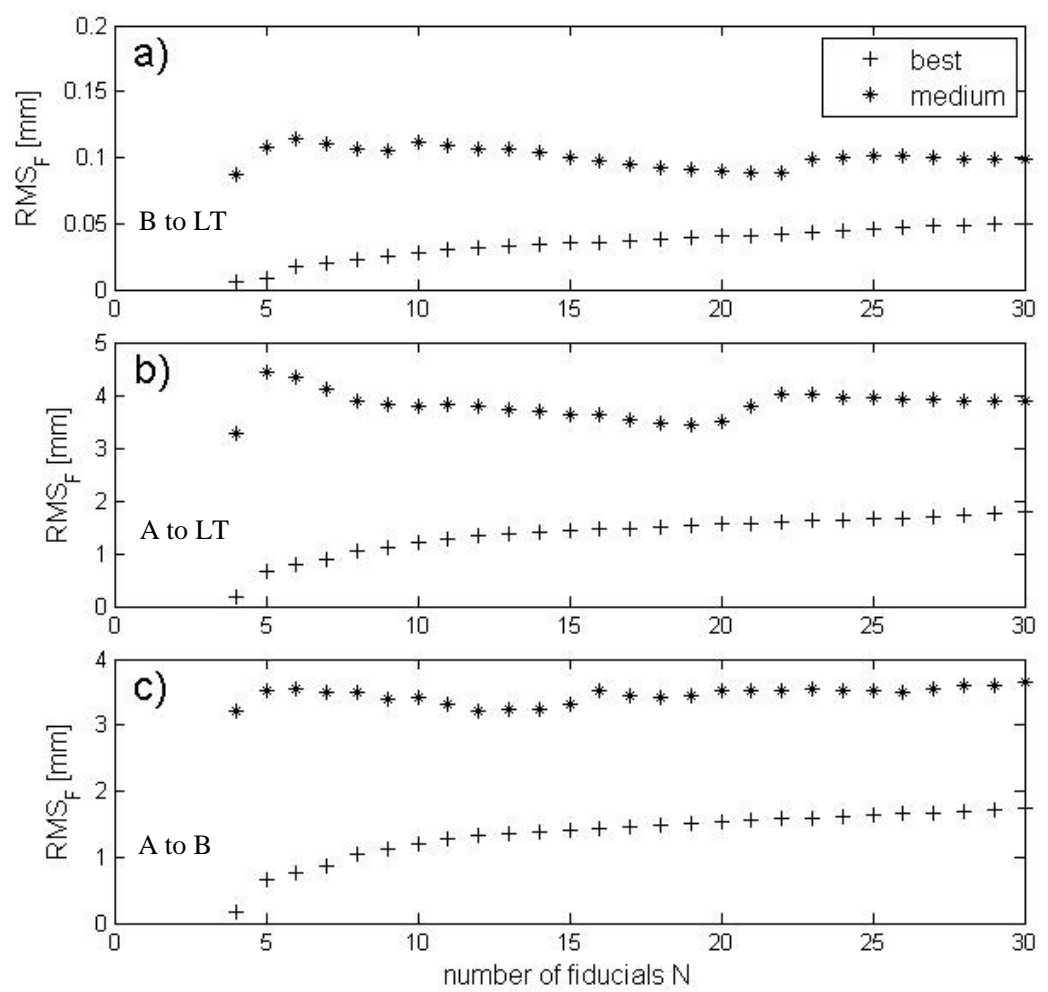

Figure 4.17. $R M S_{F}$ versus the number of fiducials $N$ used to register: a) System B to LT; b) System A to LT; c) System A to B. Two criteria for selecting the best registration were used for each combination of $N$ : based on the smallest $R M S_{F}(+)$ and medium $R M S_{F}\left(^{*}\right)$ from the middle of a sorted $R M S_{F}$ list. 

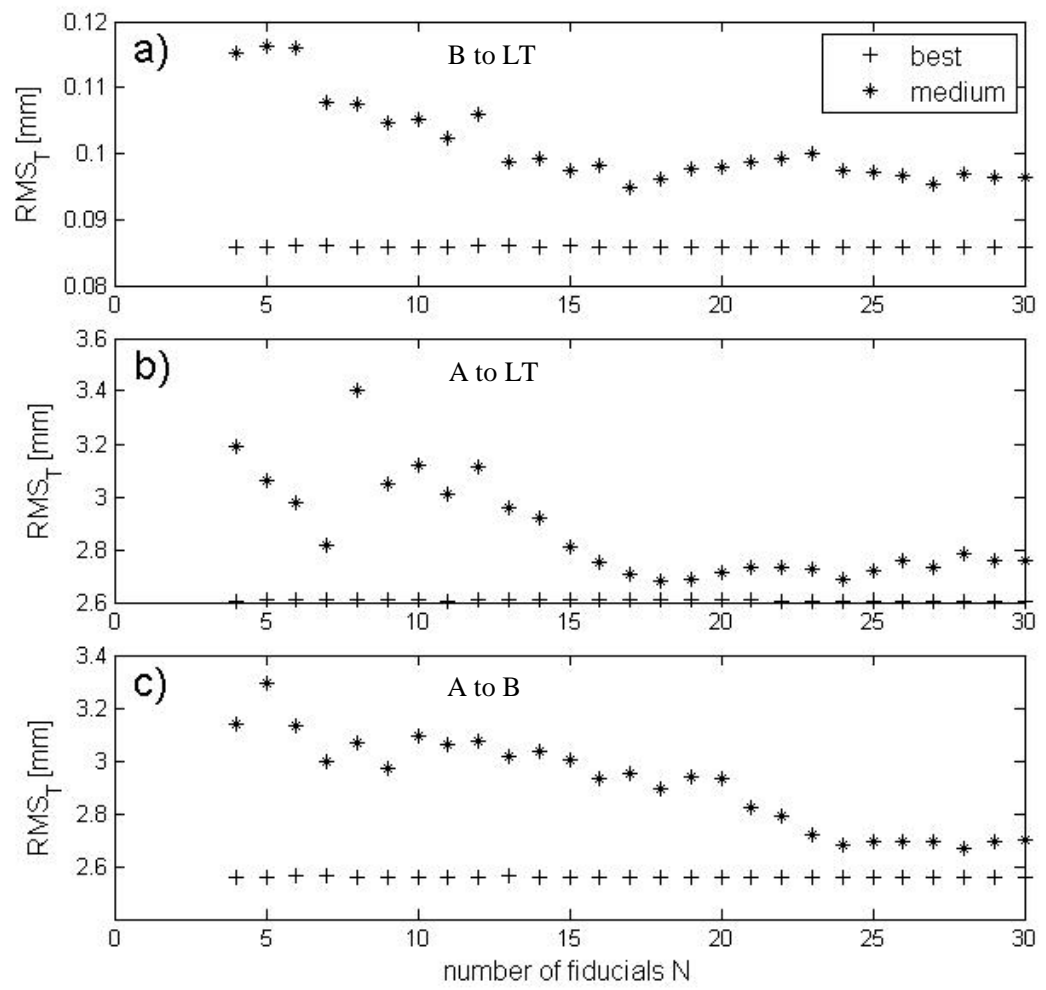

Figure 4.18. $R M S_{T}$ versus the number of fiducials $N$ used to register: a) System B to LT; b) System A to LT; c) System A to B. Two criteria for selecting the best registration were used for each combination of $N$ : based on the smallest $R M S_{T}(+)$ and medium $R M S_{T}\left({ }^{*}\right)$ from the middle of a sorted $R M S_{T}$ list.

In Figure 4.19 the smallest index $W$ versus the number of fiducials $N$ is plotted. The procedure to select the best registration gauged by $W$ is the same as described above except that this time only the smallest $W$ is plotted. 

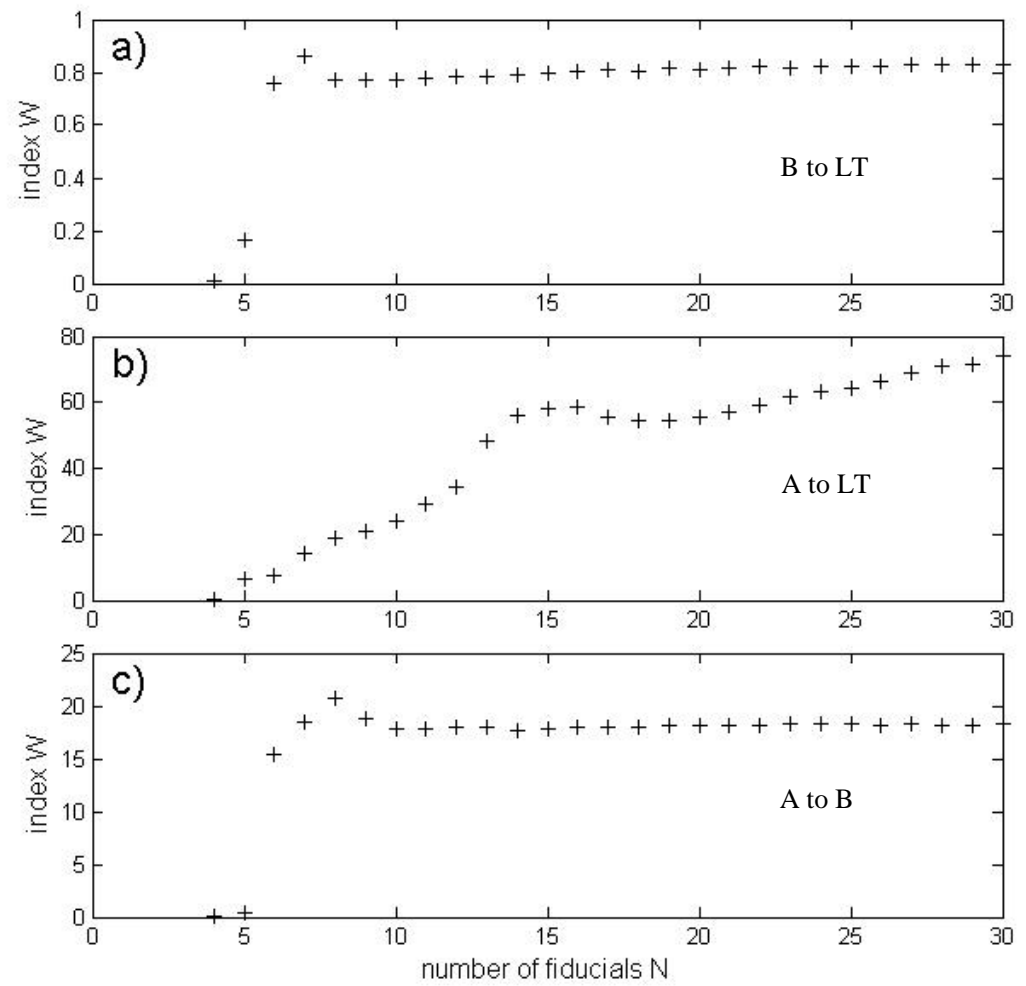

Figure 4.19. Index $W$ versus the number of fiducials $N$ used to register: a) System B to LT; b) System A to LT; c) System A to B. For each $N>4$, the smallest index $W$ from the remaining $M-N$ possible registrations is selected.

Thus far, the presented results were obtained mainly by using the averaged values (over 200 repeats) of fiducial and test points, i.e., using method 1. Figure 4.20 shows the results for $R M S_{T}$ calculated using method 2. For each set of $N$ fiducials, 200 registrations were determined (one for each repeat) together with the corresponding $R M S_{T}$. The mean and standard deviations of $R M S_{T}$ are plotted in Figure 4.20 as a function of $N$. The first combination for $N=4$ is the same as the combination of four fiducials used in Figure 4.18, each consecutive added $N+1$ fiducial was selected from the set of remaining $M-N$ fiducials to yield the smallest mean $R M S_{T}$. 

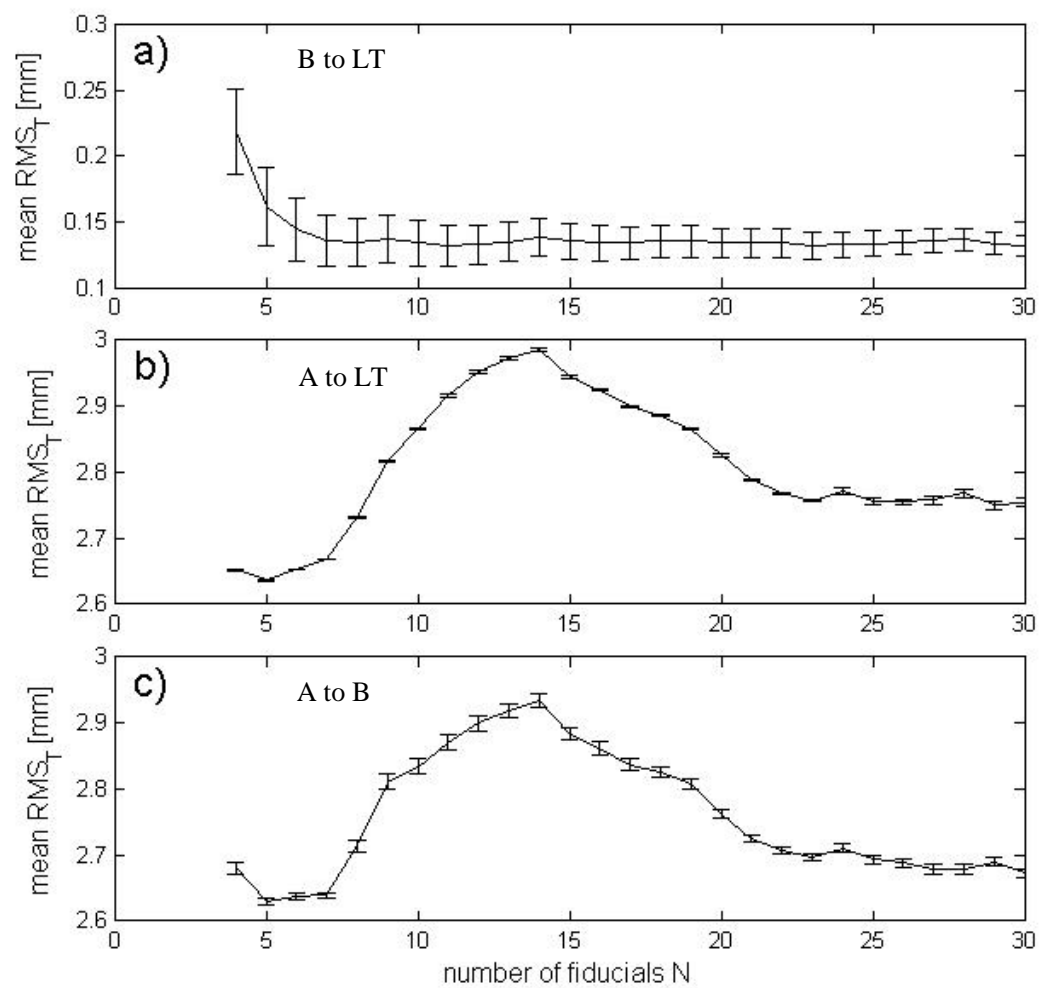

Figure 4.20. Mean $R M S_{T}$ (calculated using method 2) versus the number of fiducials $N$ used to register: a) System B to LT; b) System A to LT; c) System A to B. Error bars indicate \pm one standard deviation of $R M S_{T}$ obtained from 200 repeated measurements. 


\section{DISCUSSION}

\subsection{NOISE}

In Figure 4.1 and Table 4.1, the differences between noise magnitude $\sigma_{0}$ as defined in Eq. (1) for System A and B are shown. Generally, System A has a smaller noise level but the histogram of $\sigma_{0}$ shows that System A has more large outliers than System B. Spatial locations of these large outliers are randomly scattered in the work volume. For System B, the variation in the magnitude of $\sigma_{0}$ is smaller but there is a pattern in the locations of points with larger $\sigma_{0}$ : noise increases as the points get closer to the floor, as shown in Figure 4.1b. Therefore, whenever the instrument's noise characterization is known, selection of fiducials at locations with large $\sigma_{0}$ should be avoided if possible to ensure good registration.

Figure 4.2 provides a direct insight into the point clouds obtained from the 200 repeated measurements of the same point. It is obvious that the large outliers for $\sigma_{0}$ for System $\mathrm{A}$ are a result of some ambiguity in the system's data acquisition causing it to detect the same point in two locations, as clearly demonstrated in Figure 4.2b. This ambiguity may be the result of "lesser" coverage of the location by the system's sensors. Depending on the arrangement of the sensors, some locations may be covered by all of the sensors and some by only the minimum number of sensors. Looking at the locations of the large noise levels for System A in Figure 4.1a - towards the edges of the work volume, it appears that this may be the case.

\subsection{BIAS}

Figure 4.3 shows that the error $L$, the error of the distance between two points, is strongly correlated with the magnitude of the distance $D$ between the two points. Results shown in Figure 4.3 and Figure 4.4 clearly indicate that the rigid body assumption is reasonable for System B and incorrect for System A. Recall that the distance $d_{i j}$ used in Eq. (2a) is between the mean locations of fiducials $i$ and $j$, averaged over 200 repeats (calculated using method 1). Thus, large values of $L$ or $p$ cannot be attributed to random variations in the measurements but to systematic bias of the instrument. The presence of such large bias in the data acquired by System $A$ is expected to adversely affect the quality of the registration of System A to LT or to B. As described in Section 2, we followed the manufacturer's calibration procedure which set the necessary parameters for the entire network of cameras. However, there was no process or means to re-calibrate the individual cameras. 


\subsection{PERFORMANCE METRICS FOR REGISTRATION}

\subsubsection{Index $Q$}

Figure 4.5 shows histograms of the proxy index $F$ and index $Q$ for all $M_{N}$ combinations of $N=4$

fiducials used for registration. Based on the presence of bias, one can expect the best registration to be between System B and LT. The comparison of Figure 4.5(d, e) shows that bias degrades the quality of registration as gauged by index $Q$. Further, Figure 4.5(e, f) show that the noise in the presence of bias degrades the quality of registration much more. Proxy index $F$ has very similar distributions for all three pairs of instruments: (B, LT), (A, LT), and (A, B) and does not give any indication of the presence of bias. Again, it should be pointed out that no actual registration is performed to calculate index $F$, thus the differences between distributions of $F$ and $Q$ for the three pairs of instruments are expected. Since proxy index $F$ depends on the noise magnitude $\sigma_{0}$, Eq. (20), it is not surprising that proxy $F$ has the smallest spread for System A registered to LT, as shown in Figure 4.5b, and largest for System A to B.

Based on the histograms of index $Q$ shown in Figure 4.5(d, e), it appears that poor registration, as revealed by large values of $Q$, is caused solely by instrument bias (note that System $B$ has no bias but its instrument noise is larger than for System A, as shown in Figure 4.1 and Table 4.1). The combined effect of noise and bias significantly decreases the quality of registration as seen by the large $Q$ values in Figure $4.5 f$.

\subsection{2 $R M S_{F}$ and $R M S_{T}$}

It can be shown (see Appendix B) that for data with bias, $R M S_{F}$ must be larger than $\min R M S_{F}$ given by Eq. B1 which depends on magnitude of the bias in the acquired data; a larger bias results in a larger minRMS $S_{F}$. Therefore, the histogram of $R M S_{F}$ for System A (Figure 4.7d ) with a large bias is shifted to the right as compared to the histogram for System B (Figure 4.7a).

Histograms of the RMS shown in Figure 4.7 and calculated using methods 1 and 2 substantiate the finding of poorer registration performance for System A to LT due to bias. The peak values of $R M S_{F}$ and $R M S_{T}$ for (B to LT) registration calculated using method 1 (solid line in Figure 4.7) are an order of magnitude smaller than the corresponding values for System A (with bias) to LT. In Figure $4.7(\mathrm{~d}-\mathrm{f})$, the difference between the solid and dotted lines, i.e., effect of noise, is very slight and is not visible in the plot. Also, the spreads of $R M S_{F}$ and $R M S_{T}$ for (B to LT) are an order of magnitude smaller then corresponding spreads for (A to LT) and (A to B) registrations.

$R M S_{F}$ is frequently used to report the quality of registration and a common belief is that this metric can be directly used to estimate the important but usually not available $R M S_{T}$ (test points are usually not measured in the interest of time or because it is not possible and thus $R M S_{T}$ cannot be calculated). However, $R M S_{T}$ is more relevant to the user since knowing the error in the location of other points, such as the location of an object in the work volume, is more important than the error in the location of the fiducials. Basically, fiducials are used for 
registration and are not used otherwise. Other researchers have found that there is no correlation between $R M S_{F}$ and $R M S_{T}$ as was found in this study too [23].

Note that the spread of $R M S_{F}$ is larger than the spread of $R M S_{T}$. Other researchers have found that the distribution of $R M S_{F}$ does not depend on the location of the fiducials but only the magnitude of the noise of the fiducials. They also found that the distribution of $R M S_{T}$ depends on the locations of the targets (equivalent to test points as used in this report) relative to the fiducials. These observations were based on data with noise but with no bias [4, 9]. However, the data in Figure 4.7 has bias but no noise, and it is important to determine the relationship between of $R M S_{F}$ and $R M S_{T}$. Therefore, the ratio $s\left(R M S_{T} / R M S_{F}\right)$ is calculated for all $M_{N}$ combinations of $\mathrm{N}$-fiducials. Regardless of the magnitude of the noise or the presence of bias, $s$ appears to be greater than one for a majority of $M_{N}$ combinations of $N$-fiducials used for registration, as shown in Figure 4.7(c, f). That is, $R M S_{F}$ is smaller than $R M S_{T}$ for most cases and this is to be expected as the registration process minimizes $R M S_{F}$. The histogram of $s$ for System B (virtually no bias, better registration performance) to LT shows that almost $25 \%$ of all $M_{N}$ combinations have $R M S_{T}>2 R M S_{F}$, as shown in Figure 4.7c; similarly for System A to LT (Figure 4.7f) $R M S_{T}>1.5 R M S_{F}$. Histograms of the ratio $s$ suggest that $R M S_{F}$ should be used as a lower bound of the expected $R M S_{T}$ and a conservative estimate of $R M S_{T}$ is $R M S_{T} \approx 2 R M S_{F}$.

Comparison of the results obtained using methods 1 and 2 (solid and dotted lines in Figure 4.7) leads to an interesting observation. The peaks of the dotted lines (combined bias and noise, method 2) in Figure 4.7(a, b) are shifted to the right, larger values of $R M S_{F}$ and $R M S_{T}$ compared to the peaks of the solid lines (bias only, method 1 ). This is expected and it agrees with the previous observations made with index $Q$ that the combined presence of bias and noise degrades the quality of registration (good registration is expected to result in index $Q$ close to 1 ). Note that the error $L$ in Eq. (2a) is used to check how well the rigid body assumption holds. Large error $L$ indicates that the rigid body assumption is violated. For System $B$, the error $L$ is smaller when calculated for averaged locations of fiducials (method 1) than for single instantaneous measurement of fiducials (method 2). However, when it comes to the ratio $s, R M S_{T} / R M S_{F}$, shown in Figure 4.7c, the range of $s$ decreases for method 2 when compared with the corresponding range for method 1 . This behavior seems counter to the behavior shown in Figure 4.7(a, b) where the histograms for method 2 are shifted to the right.

For System A, the shift between the dotted and solid lines is not visible in Figure 4.7(d, e). This is because the violation of the rigid body assumption for System $\mathrm{A}$ is mostly attributed to bias and therefore method 2 yields almost identical results as method 1.

Since $s$ is dimensionless, a direct comparison between registration of B to LT and A to LT (Figure 4.7(c, f)) is possible. The dotted curve in Figure 4.7c is very similar to the solid curve in Figure 4.7f. This similarity points to the equivalence between the registration based on averaged fiducials measurements (obtained with a biased instrument which has small noise) and the registration based on a single measurement of the fiducials with a noisy but unbiased instrument. 
That is, the effect on ratio $s$ for an instrument with small noise and large bias is similar to the effect for an instrument with large noise and small bias. For A to LT registration (Figure 4.7(d f)), the shape of the dotted and solid lines are almost identical and there is no shift in the peak location as seen for B to LT registration (Figure 4.7(a, b)). This can be expected because the bias in System A is two orders of magnitude larger then instrument noise (see Table 4.1, Figure 4.3 and Figure 4.4a).

\subsubsection{Index $W$}

Histograms of index $W$ shown in Figure 4.8 also indicate that biased data has a significant effect on the quality of registration. Recall that index $W$ is the median of all $w_{k}$ defined in Eq. (18c). Each $w_{k}$ gauges how large the distance $d_{k}$ is between the transformed test point $\tilde{\boldsymbol{t}}_{k}$ and the same point $\boldsymbol{g}_{k}$ measured in the reference frame relative to the standard deviation of $d_{k}$. For noisy but unbiased data, $w_{k}$ should be less than one for $67 \%$ of the time. Values of $w_{k} \gg 2$ correspond to the situation when $\tilde{\boldsymbol{t}}_{k}$ is far away from $\boldsymbol{g}_{k}$, separated by a distance much larger than the combined uncertainties of both points. Figure 4.8a shows that for the majority of $M_{N}$ combinations of $N=4$ fiducials used to register System B to LT, the majority of reference points $\boldsymbol{g}_{k}$ are within one standard deviation of the combined uncertainty of the corresponding transformed point $\tilde{\boldsymbol{t}}_{k}$ as given by Eq. (18). In contrast, Figure 4.8(b, c) show that the uncertainty of $\tilde{\boldsymbol{t}}_{k}$ does not provide a useful estimate of its distance to $\boldsymbol{g}_{k}$ when the data contains a bias. The presence of a smaller peak at $W \approx 40$ in Figure $4.8 \mathrm{~b}$ is not clear. The spread of index $W$ in Figure 4.8c is expected to be smaller than the spread in Figure 4.8b because LT has much smaller noise than System B; thus, the corresponding $\operatorname{var}\left(d_{k}\right)$ in Eq. (18c) for LT are smaller causing $w_{k}$ to be larger.

\subsubsection{Correlation between metrics}

All five metrics discussed so far, index $Q, W, R M S_{F}, R M S_{T}$, and proxy index $F$, share one common property: a smaller numerical value corresponds to a better registration. There are, however, substantial differences between them:

- $\quad Q, W$, and $F$ are dimensionless

- $R M S_{F}, R M S_{T}$ have units of length

- $\quad Q, W, F$, and $R M S_{T}$ are based on test points while $R M S_{F}$ is based on fiducials only

- $F$ does not require registration while the remaining four can be evaluated only after registration transformation is determined.

The metrics gauge different aspects of the registration procedure and the important question arises as to how these different metrics correlate with each other. For example, if for a given combination of $N=4$ fiducials which yields the smallest $R M S_{F}$, would the registration be deemed good when gauged by index $Q$ ? 
Results shown in Figure 4.9 attempt to address this question for the registration of System B to LT. All possible $M_{N}$ combinations of fiducial placements were checked and for each metric the $m^{\text {th }}$ combination which yielded the smallest value of the metric was stored, i.e., $m(F)$ combination yielded the smallest proxy $F, m(Q)$ yielded the smallest index $Q, m\left(R M S_{F}\right)$ the smallest $R M S_{F}$, and $m\left(R M S_{T}\right)$ the smallest $R M S_{T}$. Note that the combination yielding $m(F)$ is not the same combination which yields $m(Q)$, etc. In Figure 4.9(a - e), all four metrics $Q, W, R M S_{F}$, $R M S_{T}$, and proxy index $F$ are plotted for the selected five best combinations of $N=4$ fiducials: $m(Q), m(W), m\left(R M S_{F}\right), m\left(R M S_{T}\right)$ and $m(F)$. When index $Q$ is minimum (Figure 4.9b), combinations $m(F)$ and $m\left(R M S_{T}\right)$ yield almost equally good registration (i.e., low $Q$ values) as the combination of fiducials $m(Q)$ corresponding to the smallest $Q$. However, combinations of fiducials $m(W)$ and $m\left(R M S_{F}\right)$ yielded high $Q$ values (i.e., poor registration). On the other hand, when $R M S_{F}$ is important and the best configuration of fiducials is given by $m\left(R M S_{F}\right.$ ) (Figure 4.9d), then combinations $m(F)$ and $m\left(R M S_{T}\right)$ should be avoided because they yield relatively large $R M S_{F}$; as the $R M S_{F}$ values for $m(F)$ and $m\left(R M S_{T}\right)$ are in the right tail section of the solid line in Figure 4.7a. Since $W$ and $R M S_{T}$ both depend on the distance $d_{k}$ (Eq. 18a), one may expect that $W$ and $R M S_{T}$ would be correlated. However, this is not the case as seen in Figure 4.9c, Figure 4.10c and Figure 4.11c. This is because $W$ is affected by both noise and bias while $R M S_{T}$ (as calculated in this report using method 1 ) is only affected by bias. So, based on results of registering system B to LT, there is no combination of $N=4$ fiducials which would result in all four metrics $Q, W, R M S_{F}, R M S_{T}$ having the smallest possible values simultaneously.

While there is no one combination which optimizes all four metrics, there may be some combinations for which two of the metrics are correlated. For example, if $R M S_{T}$ is the most important aspect of registration, the $m(Q)$ combination yields a $R M S_{T}$ value that is almost the same as the $m\left(R M S_{T}\right)$ combination, as shown for all three pairs of registered instruments in Figure 4.9e, Figure 4.10e, and Figure 4.11e.

Results shown in Figure 4.10 and Figure 4.11 for System A registered to LT and System A to B lead to similar conclusions. The major difference between these two figures and Figure 4.9 are much larger values of index $Q$ and $W$. However, since the spreads of $Q$ and $W$ in Figure 4.5(e, f) and Figure 4.8(b, c) are larger than for B to LT, the relative differences between four metrics are comparable.

At this point, two different strategies for finding the best placement for fiducials are possible:

1) select the metric which is the most important in a given application and use the corresponding combination of fiducials [for example: if $R M S_{T}$ is the most important, then use combination $m\left(R M S_{T}\right)$ ]

2) do not use the individual metrics and find a compromise solution: a combination of fiducials $m_{\text {all }}$ which minimizes the error function $\operatorname{err}(m)$

$$
\operatorname{err}(m)=\left\|\boldsymbol{G}^{*}-\boldsymbol{G}(m)\right\|^{2},
$$




$$
\boldsymbol{G}(m)=\left[Q(m), W(m), R M S_{F}(m), R M S_{T}(m)\right]
$$

and $\boldsymbol{G}^{*}$ is a vector whose components are the smallest possible values for the four metrics

$$
\boldsymbol{G}^{*}=\left[Q_{\text {min }}, W_{\text {min }}, R M S_{F(\min )}, R M S_{T(\min )}\right] .
$$

\subsubsection{Selection of fiducials}

Figure 4.12 shows that the proxy index $F$ may be used to select the best placement of fiducials when the quality of registration is gauged by index $Q$. The differences between the black and gray bars are small as can be concluded by comparing with the spreads of $Q$; see the corresponding histograms of $Q$ in Figure 4.5(d - f). Recall that the calculation of proxy index $F$ does not require performing the actual registration and thus, one-to-one correspondence is not expected between the elements of the sorted list of $F$ and $Q$. Nevertheless, the ranks $k_{Q}$ of sorted $Q$ corresponding to the ten smallest values of proxy $F$ are still relatively small when comparing to the total number of combinations $M_{N}=9691375$. The combinations of $N=4$ fiducials which

result in the ten smallest proxy index $F$ also result in small values of $Q(99.68 \%$ of all $Q$ 's have larger values).

The procedure proposed in this report for determining the locations of fiducials for the best possible registration requires two input parameters: size/shape of the work volume and noise characteristics of instrument(s) used to acquire the 3D data. It is important to distinguish between two possible scenarios: when the instrument noise in the work volume is variable (i.e., different noise magnitude $\sigma_{0}$ at different points) or when the noise is nearly constant and well approximated by the averaged value, $\sigma_{a v g}$, for the whole work volume. Figure 4.13 shows that the two scenarios will lead to different selection of the fiducials' locations in the same work volume. While the differences between indices $Q$ calculated for the two scenarios 1.35 and 1.33 vs. 1.74 and 1.64 (Figure 4.13a, c vs. b, d) seem to be small (approximately 0.4 ), they should be compared with the range of $Q$ (approximately 2) in Figure 4.5d, i.e., the difference in $Q$ is significant. Therefore, ignoring spatial dependence of instrument noise and replacing it with a fixed value may lead to a relatively large degradation in the quality of registration as gauged by $Q$. It should be noted that the effort to obtain the measurements throughout the work volume and to obtain many (200) repeats, such as presented in this research study, is not necessarily required before each registration for many practical situations. For example, if instrument noise depends on the distance from the sensor, it is sufficient to determine this dependence once and reuse this relationship later when determining the locations of fiducials.

\subsubsection{Effect of additional fiducials}

The discussion thus far has been on the results for a fixed number of fiducials, $N=4$. Using more fiducials for registration would presumably improve the registration but for practical applications, it may not be feasible as it is more labor intensive. The results shown in Figure 
4.14 and Figure 4.15 clearly indicate that when the initial combination is chosen such that it minimizes index $Q$ or proxy index $F$ then the additional $5^{\text {th }}$ fiducial only marginally improves the registration (cases 1, 2 in Figure 4.14 and cases 1-3 in Figure 4.15). Also, when the starting combination of $N=4$ fiducials is far from optimal, then the addition of an optimally chosen $5^{\text {th }}$ fiducial (out of the remaining $M-N$ locations) results in a large improvement in the registration performance (cases 3, 4 in Figure 4.14 and cases 4, 5 in Figure 4.15).

The influence of more fiducials on $Q, R M S_{F}$, and $R M S_{T}$ are shown in Figure 4.16 to Figure 4.18. In these figures, two starting combinations of fiducials were used - best or optimum combination and a medium combination for a given metric. The medium scenario represents the situation when the optimum combination is not used or is not possible and a combination less than optimal is chosen. The results indicate that adding more fiducials is not always beneficial. This observation depends on: which performance metric is used to gauge the improvement, the starting combination of $N=4$ fiducials, and the strategy to select the $N+1$ fiducial. When the starting combination is optimized (the "best" combination, + symbol) adding extra fiducials is only marginally beneficial at best. However, when the "medium" starting combination (* symbol) is used, the conclusion is different. For example, for the "best" combination, index $Q$ in Figure 4.16 (+ symbol) shows slight improvement, $R M S_{T}$ in Figure 4.18 is almost independent of $N$, and $R M S_{F}$ in Figure 4.17 is slowly increasing with $N$. However, for the "medium" (* symbol) combination, index $Q$ shows a large decrease for $\mathrm{N}<10$ in Figure 4.16(a, c) and a non-monotonic decrease with $N$ in Figure $4.16 \mathrm{~b}$ - a large increase for $N=14,15$ is observed, $R M S_{T}$ in Figure 4.18 shows non-monotonic decrease, $R M S_{F}$ in Figure 4.17 is almost independent of $N$. As seen in Figure 4.18, the difference between the "best" and "medium" $R M S_{T}$ for $N=4$ is about $0.04 \mathrm{~mm}, 0.6 \mathrm{~mm}$, and $0.6 \mathrm{~mm}$ for B to LT, A to LT, and A to B, respectively. This difference for $R M S_{F}$ is about $0.8 \mathrm{~mm}, 3 \mathrm{~mm}$, and $3 \mathrm{~mm}$ (Figure 4.17) for $N=4$ for B to LT, A to LT, and A to B, respectively. Depending on the application, it may be important that effort be invested in selecting the optimum combination.

As mentioned previously, for $N>4$ the total number of $N$-combinations $M_{N}$ increases rapidly with $N$, and it is impossible to check all $M_{N}$ combinations and select the one which yields the smallest $Q$. The strategy of retaining the best $N$ fiducials and adding $N+1$ fiducials from the remaining $(M-N)$ fiducials explores only a tiny fraction of all $M_{N}$ combinations. Thus, it is not possible to state with complete certainty that such selected combinations of $N+1$ fiducials will yield the smallest possible $Q$. An attempt was made to increase the number of combinations checked. For case 3 shown in Figure 4.15, the first $10^{6}$ combinations yielding the smallest index $F$ from the ordered list were checked and for each of them, the $5^{\text {th }}$ fiducial yielding the smallest $F$ from the remaining $(M-N)$ fiducials was selected. Finally, the combination of $N=5$ fiducials which yielded the smallest $F$ out of $10^{6}$ combinations was selected. Data for case 2 and 3 in Figure 4.15 indicate that the method of adding $N+1$ fiducial to the previous best $N$-fiducials may be good enough and the obtained index $Q(N+1)$ is close to the global minimum. Also, the values of index $Q$ for cases 1 and 2 in Figure 4.14 and Figure 4.15 support the claim that proxy index $F$ 
(which can be quickly calculated) may be used instead of $Q$ while searching for the best combinations of fiducials. This claim is also supported by the data shown in Figure 4.12.

The index $W$ plotted in Figure 4.19 also negates the common belief that using more fiducials for registration is always beneficial. In fact, $W$ remains less than one when registering System $B$ to LT (Figure 4.19a) and greater than one for System A registered to B or to LT (Figure 4.19(b, c)) when $N>5$. Thus, good registration $(W<1)$ for $N=4$ remains good and bad registration remains bad regardless of the number of fiducials $N$ when registration performance is gauged by index $W$.

The same strategy of adding $N+1$ fiducial to the best $N$-fiducials was applied to the mean $R M S_{T}$ calculated using method 2 from 200 repeats (i.e., 200 registrations for nominally the same combination of $N$-fiducials). Certain improvement is observed in Figure 4.20a only for $N \leq 6$ for registering System B to LT. For $N>6$, adding extra $N+1$ fiducial is either not beneficial or yields results worse than the starting mean $R M S_{T}$ for $N=4$, as evident from Figure 4.20(b, c). Note that the starting values of mean $R M S_{T}$ for $N=4$ calculated using method 2 are higher than the corresponding values of $R M S_{T}$ calculated by method 1, as shown in Figure 4.18 and Figure 4.20. This is expected as averaged locations of fiducials obey the rigid body assumption better than individual noisy locations. 


\section{FINAL REMARKS}

In this report, some guidelines for selecting the locations and number of points for registering a working coordinate frame to a reference coordinate frame are presented. The proposed procedure requires a priori knowledge of the instrument's noise and the size/geometry of the work volume. If the spatial distribution of instrument noise is unknown, the selection of fiducials for the registration will be based solely on the size and shape of the work volume and may lead to a set of fiducials which may not yield the best possible registration.

To select the fiducials for registration, an index $F$ was proposed, and the results show that this index may be used as a proxy for index $Q$. Index $F$ is based on the placement of the fiducials and instrument noise. It does not require the calculation of the registration transformation $\{\boldsymbol{\Omega}, \boldsymbol{\tau}\}$ (as required for index $Q$ ) and can therefore be used to rapidly calculate index $F$ for many different combinations of fiducials to yield an optimum fiducial selection. Since index $F$ is correlated with $Q$, the uncertainty of the transformed point is minimized for small $F$ but that does not necessarily mean the $R M S_{T}$ is also minimized.

The uncertainty of a transformed test point in the reference frame will always be equal to or greater than the uncertainty of the test point in the working frame. The transformed uncertainty is only equal to the uncertainty of the point in the working frame for an ideal registration, i.e., fiducials without bias and noise. In a realistic scenario, the uncertainty of the transformed test point will be larger than the uncertainty of the point in the working frame, and the uncertainty of the transformed point may be estimated by multiplying the uncertainty of the point in the working frame by index $Q$.

If $J$ repeated measurements of the fiducials are obtained, two processing strategies are possible: 1) calculate the average locations of the fiducials, perform one registration, and then apply the transformation parameters, 2) perform $J$ registrations, average the $J$ registration parameters, and then apply the average transformation parameters. The first strategy is recommended because it results in smaller $R M S_{F}$ and $R M S_{T}$.

The concept of ground truth does not apply to the six registration parameters because these parameters cannot be measured with another (more accurate and precise) instrument. Therefore, four metrics, which were proposed to quantify the performance of the registration, evaluate the systematic and the random errors (or the accuracy and the precision) based on sets of points acquired in both frames (fiducials and test points). This study shows that no single metric from the proposed four metrics can adequately describe the overall performance of registration. The optimization of different metrics yields different locations of fiducials. The choice of metric depends on the information available about the instrument and the application (for example, a priori knowledge of the spatial distribution of instrument noise or the magnitude of the bias). 
In practice, test points are usually not available since all points acquired in both coordinate frames are used as fiducials for registration and the effort to measure additional points (test points) is not made in the interest of time or is not possible. Therefore, only the root mean square for fiducials $\left(R M S_{F}\right)$ can be evaluated and is frequently used as a substitute for the root mean square for test points $\left(R M S_{T}\right)$. As mentioned in Section 1, studies [12], [15-19] have found that there is no correlation between $R M S_{F}$ (FRE) and $R M S_{T}$ (TRE), and the data in this study also support this finding. However, in the absence of any other data, a conservative estimate of $R M S_{T}$ is suggested. This study shows that $R M S_{F}$ should be used as a lower bound for $R M S_{T}$ and that it is generally more conservative to assume $R M S_{T} \geq 2 R M S_{F}$.

A check of the rigid body assumption should also be performed as outlined in Sections 3.2 and 4.2. Large values of the dimensionless ratio $p(p>3)$ indicate the presence of a large bias in the data. Uncompensated instrument bias adversely affects the quality of registration as gauged by index $Q, R M S_{T}, R M S_{F}$, and $W$. Therefore, if possible, it is recommended that the instrument be calibrated to remove any bias.

With regards to how many fiducials to use for registration, the use of a larger number of fiducials $(N>4)$ is either marginally beneficial or not necessary when the starting combination with $N=4$ fiducials is optimally selected. When the optimal starting combination cannot be determined, adding extra fiducials usually leads to mixed results and no generalizable conclusions could be formulated about when to expect improved or worse registration.

\subsection{FUTURE WORK}

In [4], it was proposed that a test point located at the centroid of the fiducials would result in the minimum $R M S_{T}$ (TRE). However, later studies [19, 22] have shown that this may not necessarily be true. In future work, we will investigate the discrepancy between the analytical expression for $R M S_{T}$ (TRE) in [4] and experimental results by [19, 22]. Also, the issue of bias is important as bias may be present in field conditions and may not be easily eliminated. We will also address methods to reduce the bias from the measured 3D data. 


\section{APPENDIX A}

In this Appendix, following the general framework provided in [7, 24], the explicit form of the covariance matrix $\boldsymbol{C}_{\boldsymbol{\Theta}}$ used in Eq. (13) and the Jacobian $\boldsymbol{J}_{\boldsymbol{t}}$ defined in Eq. (14) are provided. The rotation matrix $\boldsymbol{\Omega}$ in (axis, angle) representation is defined as

$$
\boldsymbol{\Omega}(\vartheta, \varphi, \rho)=\cos \rho \boldsymbol{I}+\sin \rho\left[\begin{array}{ccc}
0 & -a_{z} & a_{y} \\
a_{z} & 0 & -a_{x} \\
-a_{y} & a_{x} & 0
\end{array}\right]+(1-\cos \rho) \boldsymbol{a}^{T} \boldsymbol{a},
$$

where the unit row vector $\boldsymbol{a}(\vartheta, \varphi)$ is the axis of rotation parametrized as in Eq. (15) and $\boldsymbol{I}$ is the identity matrix. A gradient column vector $\boldsymbol{G}$ of the error function $h$ from Eq. (5) with respect to the registration parameters $\boldsymbol{\Theta}=\left(\vartheta, \varphi, \rho, \tau_{x}, \tau_{y}, \tau_{z}\right)$ can be written as

$$
\boldsymbol{G}(\boldsymbol{X}, \boldsymbol{\Theta})=\left(G_{\vartheta}, G_{\varphi}, G_{\rho}, G_{\tau_{x}}, G_{\tau_{y}}, G_{\tau_{z}}\right)^{T}
$$

where $\boldsymbol{X}$ is the $3 \times 2 N$ matrix $\left[\boldsymbol{Z}_{1}, \cdots, \boldsymbol{Z}_{N}, \boldsymbol{Y}_{1}, \cdots, \boldsymbol{Y}_{N}\right]$ of fiducials in the working and reference coordinate frame, respectively, and

$$
\begin{aligned}
& \left(G_{\vartheta}, G_{\varphi}, G_{\rho}\right)=\left(H\left(\boldsymbol{\Omega}_{\vartheta}\right), H\left(\boldsymbol{\Omega}_{\varphi}\right), H\left(\boldsymbol{\Omega}_{\rho}\right)\right) \\
& \left(G_{\tau_{x}}, G_{\tau_{y}}, G_{\tau_{z}}\right)=2 \sum_{n=1}^{N}\left(\boldsymbol{\Omega Z}_{n}-\boldsymbol{Y}_{n}+\boldsymbol{\tau}\right),
\end{aligned}
$$

where

$$
H(\boldsymbol{\Gamma})=2 \sum_{n=1}^{N}\left[\left(\boldsymbol{\tau}-\boldsymbol{Y}_{n}\right)^{T} \boldsymbol{\Gamma} \boldsymbol{Z}_{n}\right], \boldsymbol{\Omega}_{\alpha}=\frac{\partial \boldsymbol{\Omega}}{\partial \alpha} \text { for } \alpha=\vartheta, \varphi, \rho
$$

When the error $h$ is at its minimum, the gradient $\boldsymbol{G}$ in Eq. (A2) has to be a zero vector and the Taylor's expansion in the linear approximation yields the following:

$$
\boldsymbol{G}(\boldsymbol{X}+\Delta \boldsymbol{X}, \boldsymbol{\Theta}+\Delta \boldsymbol{\Theta})=\boldsymbol{G}(\boldsymbol{X}, \boldsymbol{\Theta})+\frac{\partial \boldsymbol{G}}{\partial \boldsymbol{X}} \Delta \boldsymbol{X}+\frac{\partial \boldsymbol{G}}{\partial \boldsymbol{\Theta}} \Delta \boldsymbol{\Theta}=\mathbf{0},
$$

where the $6 \times 2 N$ matrix $\frac{\partial G}{\partial X}$ and $6 \times 6$ matrix $\frac{\partial G}{\partial \boldsymbol{\theta}}$ are equal

$$
\frac{\partial \boldsymbol{G}}{\partial \boldsymbol{X}}=2\left[\begin{array}{cccccc}
\left(\boldsymbol{\tau}-\boldsymbol{Y}_{1}\right)^{T} \boldsymbol{\Omega}_{\vartheta} & \cdots & \left(\boldsymbol{\tau}-\boldsymbol{Y}_{N}\right)^{T} \boldsymbol{\Omega}_{\vartheta} & -\left(\boldsymbol{\Omega}_{\vartheta} \boldsymbol{Z}_{1}\right)^{T} & \cdots & -\left(\boldsymbol{\Omega}_{\vartheta} \boldsymbol{Z}_{N}\right)^{T} \\
\left(\boldsymbol{\tau}-\boldsymbol{Y}_{1}\right)^{T} \boldsymbol{\Omega}_{\varphi} & \cdots & \left(\boldsymbol{\tau}-\boldsymbol{Y}_{N}\right)^{T} \boldsymbol{\Omega}_{\varphi} & -\left(\boldsymbol{\Omega}_{\varphi} \boldsymbol{Z}_{1}\right)^{T} & \cdots & -\left(\boldsymbol{\Omega}_{\varphi} \boldsymbol{Z}_{N}\right)^{T} \\
\left(\boldsymbol{\tau}-\boldsymbol{Y}_{1}\right)^{T} \boldsymbol{\Omega}_{\varphi} & \cdots & \left(\boldsymbol{\tau}-\boldsymbol{Y}_{N}\right)^{T} \boldsymbol{\Omega}_{\rho} & -\left(\boldsymbol{\Omega}_{\rho} \boldsymbol{Z}_{1}\right)^{T} & \cdots & -\left(\boldsymbol{\Omega}_{\rho} \boldsymbol{Z}_{N}\right)^{T} \\
\boldsymbol{\Omega} & \cdots & \boldsymbol{\Omega} & -\boldsymbol{I} & \cdots & -\boldsymbol{I}
\end{array}\right]
$$




$$
\frac{\partial \boldsymbol{G}}{\partial \boldsymbol{\theta}}=2 \sum_{n=1}^{N}\left[\begin{array}{cccc}
\left(\boldsymbol{\Omega}_{\vartheta \vartheta} \boldsymbol{Z}_{n}\right)^{T}\left(\boldsymbol{\tau}-\boldsymbol{Y}_{n}\right) & \left(\boldsymbol{\Omega}_{\varphi \vartheta} \boldsymbol{Z}_{n}\right)^{T}\left(\boldsymbol{\tau}-\boldsymbol{Y}_{n}\right) & \left(\boldsymbol{\Omega}_{\rho \vartheta} \boldsymbol{Z}_{n}\right)^{T}\left(\boldsymbol{\tau}-\boldsymbol{Y}_{n}\right) & \left(\boldsymbol{\Omega}_{\vartheta} \boldsymbol{Z}_{n}\right)^{T} \\
\left(\boldsymbol{\Omega}_{\vartheta \varphi} \boldsymbol{Z}_{n}\right)^{T}\left(\boldsymbol{\tau}-\boldsymbol{Y}_{n}\right) & \left(\boldsymbol{\Omega}_{\varphi \varphi} \boldsymbol{Z}_{n}\right)^{T}\left(\boldsymbol{\tau}-\boldsymbol{Y}_{n}\right) & \left(\boldsymbol{\Omega}_{\rho \varphi} \boldsymbol{Z}_{n}\right)^{T}\left(\boldsymbol{\tau}-\boldsymbol{Y}_{n}\right) & \left(\boldsymbol{\Omega}_{\varphi} \boldsymbol{Z}_{n}\right)^{T} \\
\left(\boldsymbol{\Omega}_{\vartheta \rho} \boldsymbol{Z}_{n}\right)^{T}\left(\boldsymbol{\tau}-\boldsymbol{Y}_{n}\right) & \left(\boldsymbol{\Omega}_{\varphi \rho} \boldsymbol{Z}_{n}\right)^{T}\left(\boldsymbol{\tau}-\boldsymbol{Y}_{n}\right) & \left(\boldsymbol{\Omega}_{\rho \rho} \boldsymbol{Z}_{n}\right)^{T}\left(\boldsymbol{\tau}-\boldsymbol{Y}_{n}\right) & \left(\boldsymbol{\Omega}_{\rho} \boldsymbol{Z}_{n}\right)^{T} \\
\boldsymbol{\Omega}_{\vartheta} \boldsymbol{Z}_{n} & \boldsymbol{\Omega}_{\varphi} \boldsymbol{Z}_{n} & \boldsymbol{\Omega}_{\rho} \boldsymbol{Z}_{n} & \boldsymbol{I}
\end{array}\right]
$$

and $\boldsymbol{\Omega}_{\alpha \beta}=\frac{\partial^{2} \Omega}{\partial \alpha \partial \beta}$ for $\alpha, \beta=\vartheta, \varphi, \rho$. Then, the covariance matrix of the registration parameters $\boldsymbol{\Theta}$ can be written as

$$
\boldsymbol{C}_{\boldsymbol{\theta}}=\left(\frac{\partial G}{\partial \boldsymbol{\theta}}\right)^{-1} \frac{\partial G}{\partial X} \boldsymbol{C}_{X}\left(\frac{\partial G}{\partial X}\right)^{T}\left(\frac{\partial G}{\partial \theta}\right)^{T}
$$

where $\boldsymbol{\Gamma}^{-1}$ is the inverse matrix of $\boldsymbol{\Gamma}$ and the $2 N \times 2 N$ covariance matrix of the fiducials $\boldsymbol{C}_{\boldsymbol{X}}$ is given by

$$
\boldsymbol{C}_{\boldsymbol{X}}=\left[\mathbf{z}_{1}, \cdots, \mathbf{z}_{N}, \boldsymbol{y}_{1}, \cdots, \boldsymbol{y}_{N}\right]^{T}\left[\mathbf{z}_{1}, \cdots, \mathbf{z}_{N}, \boldsymbol{y}_{1}, \cdots, \boldsymbol{y}_{N}\right] .
$$

The equations presented thus far are general and valid for any parameterization of the rotation matrix $\boldsymbol{\Omega}$. However, the final form of the covariance matrix of the registration parameters $\boldsymbol{C}_{\boldsymbol{\theta}}$ depends on the matrices $\boldsymbol{\Omega}_{\alpha}$ and $\boldsymbol{\Omega}_{\alpha \beta}$ (for $\alpha, \beta=\vartheta, \varphi, \rho$ ) of the first and second order derivatives which, in turn, depend on the particular definition of the three angles $\{\vartheta, \varphi, \rho\}$ parameterizing matrix $\boldsymbol{\Omega}$. In the (axis, angle) representation of rotation matrix given by Eq. (A1) and a parameterization of the axis $\boldsymbol{a}(\vartheta, \varphi)$ as in Eq. (15), corresponding derivatives can be easily calculated.

Similarly (once the parametrization of the rotation matrix $\boldsymbol{\Omega}$ is selected), the Jacobian $\boldsymbol{J}_{\boldsymbol{t}}$ in Eq. (14) of the transformed $k^{\text {th }}$ test point in Eq. (12) can be calculated as

$$
\boldsymbol{J}_{\boldsymbol{t}}\left(\boldsymbol{\Theta}, \boldsymbol{t}_{k}\right)=\left[\begin{array}{lllll}
\boldsymbol{\Omega}_{\vartheta} \boldsymbol{t}_{k} & \boldsymbol{\Omega}_{\varphi} \boldsymbol{t}_{k} & \boldsymbol{\Omega}_{\rho} \boldsymbol{t}_{k} & \boldsymbol{I} & \boldsymbol{\Omega}
\end{array}\right] .
$$




\section{APPENDIX B}

\section{Theorem}

For any set of $N \geq 3$ pairs of fiducials $\left\{Z_{n}, Y_{n}\right\}$ used to calculate the registration transformation $\{\boldsymbol{\Omega}, \boldsymbol{\tau}\}$, the resulting $R M S_{F}$ is always larger or equal to $\min R M S_{F}$ defined as

$$
\operatorname{minR} M S_{F}=\sqrt{\frac{1}{2 N(N-1)} \sum_{i=1}^{j-1} \sum_{j=2}^{N} L_{i, j}^{2}},
$$

where the error $L_{i, j}$, similar to that in Eq. 2a, is the difference between two distances $y_{i, j}$ and $z_{i, j}$

$$
L_{i, j}=y_{i, j}-z_{i, j}=\left\|\boldsymbol{Y}_{i}-\boldsymbol{Y}_{j}\right\|-\left\|\boldsymbol{Z}_{i}-\boldsymbol{Z}_{j}\right\|
$$

Note that $L_{i, j}$ characterize how well the acquired data satisfy rigid body assumption and $\min R M S_{F}$ does not require that the registration transformation $\{\Omega, \tau\}$ be calculated since the distances $y_{i, j}$ and $z_{i, j}$ are invariants to rotation and translation.

\section{Proof}

$R M S_{F}$ can be written as

$$
R M S_{F}=\sqrt{\frac{1}{N} \sum_{n=1}^{N} d_{n}^{2}}
$$

where $d_{n}$ is the distance between $n^{\text {th }}$ fiducial measured in reference frame $\boldsymbol{Y}_{n}$ and the fiducial $\boldsymbol{Z}_{n}$ transformed from working frame to reference frame, $\widetilde{\boldsymbol{Z}}_{n}=\boldsymbol{\Omega} \boldsymbol{Z}_{n}+\boldsymbol{\tau}$

$$
d_{n}=\left\|\widetilde{\mathbf{Z}}_{n}-\boldsymbol{Y}_{n}\right\|
$$

Since registration transformation preserves the distance between any two points

$$
\left\|Z_{i}-Z_{j}\right\|=\left\|\widetilde{Z}_{i}-\widetilde{Z}_{j}\right\|
$$

the error $L_{i, j}$ in Eq. A13 can be calculated as

$$
L_{i, j}=\left\|Y_{i}-Y_{j}\right\|-\left\|\widetilde{Z}_{i}-\widetilde{Z}_{j}\right\|
$$

Let's consider the configuration in which all four points $\left\{\boldsymbol{Y}_{i}, \boldsymbol{Y}_{j}, \widetilde{\boldsymbol{Z}}_{i}, \widetilde{\boldsymbol{Z}}_{j}\right\}$ are collinear, see Fig. B1a. Then,

$$
r_{i, j}=d_{i}^{2}+d_{j}^{2}=\left(L_{i, j} \mp d_{j}\right)^{2}+d_{j}^{2},
$$


Where the minus sign corresponds to the configuration when the segment $\left[\boldsymbol{Y}_{i}, \boldsymbol{Y}_{j}\right]$ is inside the segment $\left[\widetilde{\boldsymbol{Z}}_{i}, \widetilde{\boldsymbol{Z}}_{j}\right]$ and the plus sign is used when both segments partially overlap (for example: $\boldsymbol{Y}_{j}$ is to the right of $\widetilde{\boldsymbol{Z}}_{j}$ ).

a)

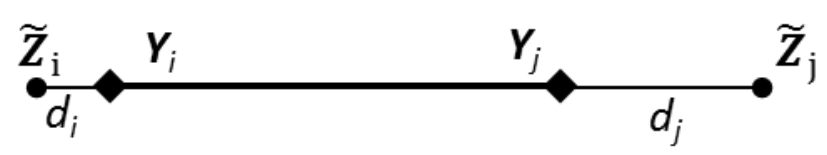

b)
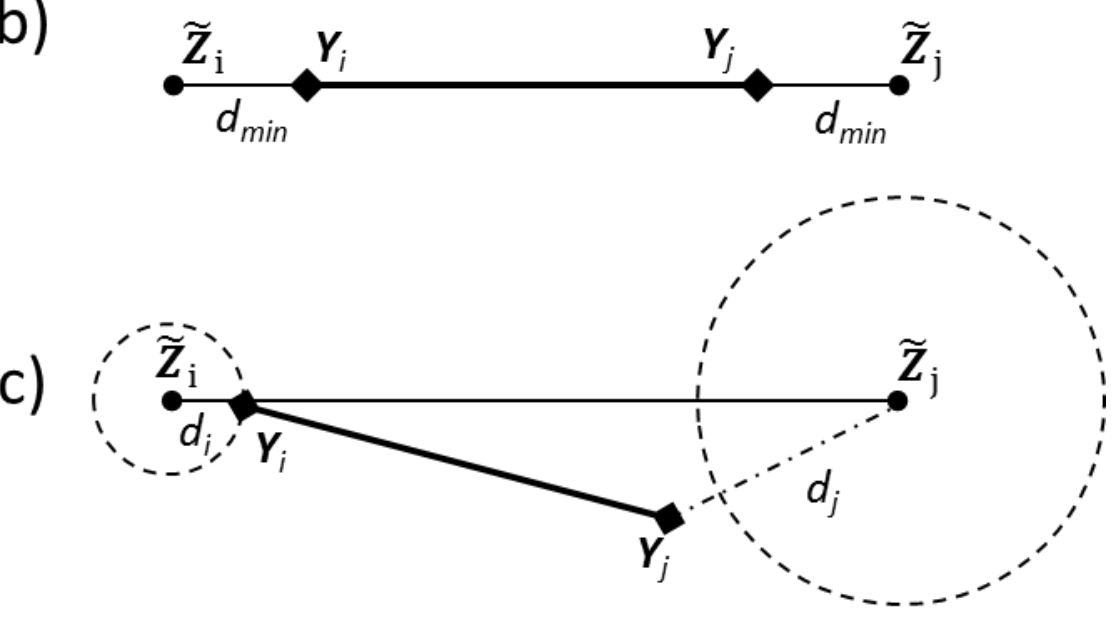

Figure B.1. Configurations of two pairs of fiducials: $\left\{\boldsymbol{Y}_{i}, \boldsymbol{Y}_{j}\right\}$ measured in the reference frame and $\left\{\widetilde{\boldsymbol{Z}}_{i}, \widetilde{\boldsymbol{Z}}_{j}\right\}$ transformed from the working to the reference frame. a) All four points are collinear, where distances $d_{i}$ and $d_{j}$ are the distance between the transformed point and the corresponding point in the reference frame; b) configuration yielding the smallest possible sum $d_{i}^{2}+d_{j}^{2}$ for given fixed distances $y_{i, j}$ and $\mathrm{z}_{\mathrm{i}, \mathrm{j}}$, see Eq. B2; c) example of a general configuration where not all four points are collinear, distance $\left\|\boldsymbol{Y}_{j}-\widetilde{\boldsymbol{Z}}_{j}\right\|$ is larger than $d_{j}$ in a). In c), the radius of the dashed circles equal $d_{i}$ and $d_{j}$ for the collinear case a).

It is easy to find that $r_{i, j}$ reaches its minimum when $\frac{\partial r_{i, j}}{\partial d_{j}}=0$. Then,

$$
d_{j}=d_{i}=d_{\min }= \pm \frac{1}{2} L_{i, j}
$$

see Figure B.1b. If only three of the four points $\left\{\boldsymbol{Y}_{i}, \boldsymbol{Y}_{j}, \widetilde{\boldsymbol{Z}}_{i}, \widetilde{\boldsymbol{Z}}_{j}\right\}$ are collinear, then one of the points (for example: $\boldsymbol{Y}_{j}$ in Fig.A.1c) has to have the distance $\left\|\boldsymbol{Y}_{j}-\widetilde{\boldsymbol{Z}}_{j}\right\|$ that is larger than $d_{j}$ in Fig. B.1a when all points were collinear. Thus, for any $(i, j)$ pairs of fiducials

$$
d_{j}^{2}+d_{i}^{2} \geq \frac{1}{2} L_{i, j}^{2} .
$$


It follows from above that

$$
(N-1) \sum_{n=1}^{N} d_{n}^{2} \geq \frac{1}{2} \sum_{i=1}^{i-1} \sum_{j=2}^{N} L_{i, j}^{2}
$$

From the definition of $R M S_{F}$ in Eq. B3 and $\min R M S_{F}$ in Eq. B1 follows that for any set of $N$ fiducials

$$
R M S_{F} \geq \min R M S_{F}
$$

$R M S_{F}$ equals $\min R M S_{\mathrm{F}}$ only for ideal registration when all $d_{n}=0$ (no noise or bias in the measurement of the fiducials in both frames). 


\section{REFERENCES}

[1] VIM, "International Vocabulary of Metrology - Basic and General Concepts and Associated Terms," JCGM 2012.

[2] M. Bennamoun and G. J. Mamic, "Chapter 3. 3-D Object Creation for Recognition," in Object Recognition London: Springer, 2002, pp. 101 - 159.

[3] J. M. Fitzpatrick and J. B. West, "The Distribution of Target Registration Error in RigidBody Point-Based Registration," IEEE Trans. Medical Imaging, vol. 20, pp. 917-927, 2001.

[4] J. M. Fitzpatrick, J. B. West, and C. R. Maurer, "Predicting Error in Rigid-Body PointBased Registration," IEEE Trans. Medical Imaging, vol. 17, pp. 694 -702, 1998.

[5] K. Kanatani, "Analysis of 3-D Rotation Fitting," IEEE TPAMI, vol. 16, pp. 543-549, 1994.

[6] C. R. Maurer, J. M. Fitzpatrick, M. Y. Wang, R. L. Galloway, R. J. Maciunas, and G.S.Allen, "Registration of Head Volume Images Using Implantable Fiducial Markers," IEEE Trans. Medical Imaging, vol. 16, pp. 447-448, 1997.

[7] X. Pennec and J. P. Thirion, "A Framework for Uncertainty and Validation of 3-D Registration Methods based on Points and Frames," International Journal of Computer Vision, vol. 25, pp. 203-229, 1997.

[8] R. Sibson, "Studies in the robustness of multidimensional scaling: Perturbation analysis of classical scaling," J. Roy. Statist. Soc. B, vol. 41, pp. 217-229, 1979.

[9] J. B. West, J. M. Fitzpatrick, S. A. Toms, C. R. Maurer, and R. J. Maciunas, "Fiducial Point Placement and the Accuracy of Point-based, Rigid Body Registration," Neurosurgery, vol. 48, pp. 810-817, 2001.

[10] M. H. Moghari and P. Abolmaesumi, "Distribution of Target Registration Error for Anisotropic and Inhomogeneous Fiducial Localization Error," IEEE Trans. Medical Imaging, vol. 28, pp. 799-813, 2009.

[11] A. Seginer, "Rigid-body point-based registration: The distribution of the target registration error when the fiducial registration errors are given," Medical Image Analysis, vol. 15, pp. 397-413, 2011.

[12] R. R. Shamir and L. Joskowicz, "Worst-case analysis of target localization errors in fiducial-based rigid body registration," in Medical Imaging 2009: Image Processing: SPIE, 2009.

[13] R. R. Shamir, L. Joskowicz, and Y. Shoshan, "Fiducial Optimization for Minimal Target Registration Error in Image-Guided Neurosurgery," IEEE Trans. Medical Imaging, vol. 31, pp. 725-737, 2012.

[14] A. D. Wiles, A. Likholyot, D. D. Frantz, and T. M. Peters, "A Statistical Model for PointBased Target Registration Error With Anisotropic Fiducial Localizer Error," IEEE Trans. Medical Imaging, vol. 27, pp. 378-390, 2008.

[15] R. Balachandran and J. M. Fitzpatrick, "The distribution of registration error of a fiducial marker in rigid-body point-based registration," in SPIE Proceedings, San Diego, CA, 2008.

[16] A. Danilchenko and J. M. Fitzpatrick, "General approach to error prediction in point registration," in Proc. of SPIE Medical Imaging: Visualization, Image-Guided Procedures, and Modeling, 2010. 
[17] M. H. Moghari and P. Abolmaesumi, "Distribution of Fiducial Registration Error in Rigid-Body Point-Based Registration," IEEE Trans. Medical Imaging, vol. 28, pp. 1791 1801, 2009.

[18] M. H. Moghari and P. Abolmaesumi, "Understanding the Effect of Bias in Fiducial Localization Error on Point-Based Rigid-Body Registration," IEEE Trans. Medical Imaging, vol. 29, pp. 1730-1738, 2010.

[19] R. R. Shamir and L. Joskowicz, "Geometrical analysis of registration errors in pointbased rigid-body registration using invariants," Medical Image Analysis, vol. 15, pp. 8595, 2011.

[20] I. J. Gerard and D. L. Collins, "An analysis of tracking error in image-guided neurosurgery," Int. J. Computer Assisted Radiology and Surgery, pp. 1-10, 2015.

[21] R. R. Shamir, L. Joskowicz, and Y. Shoshan, "What is the actual fiducial localization error in image-guided neuronavigation?," Int. J. Computer Assisted Radiology and Surgery, vol. 5, pp. S106-S113, 2010.

[22] W. Zhang, C. Wang, H. Yu, Y. Liu, and G. Shen, "Effect of fiducial configuration on target registration error in image-guided cranio-maxillofacial surgery," Journal of Cranio-Maxillo-Facial Surgery, vol. 39, pp. 407-411, 2011.

[23] J. M. Fitzpatrick, "Fiducial registration error and target registration error are uncorrelated," in SPIE Medical Imaging: Visualisation, Image-Guided Procedures and Modeling, 2009.

[24] R. M. Haralick, "Propagating covariance in computer vision," Pattern Recognition, vol. 1, pp. 493-498, 1994. 